\title{
Engineered exosomes as an in situ DC-primed vaccine to boost antitumor immunity in breast cancer
}

Lanxiang Huang ${ }^{1,2 \dagger}$, Yuan Rong ${ }^{1,2 \dagger}$, Xuan Tang ${ }^{1,2}$, Kezhen Yi ${ }^{1,2}$, Peng $\mathrm{Qi}^{3}$, Jinxuan Hou ${ }^{4}$, Weihuang Liu $^{5}$, Yuan $\mathrm{He}^{5}$, Xing Gao ${ }^{6}$, Chunhui Yuan ${ }^{2,7^{*}}$ and Fubing Wang ${ }^{1,2,8^{*}}$

\begin{abstract}
Background: Dendritic cells (DCs) are central for the initiation and regulation of innate and adaptive immunity in the tumor microenvironment. As such, many kinds of DC-targeted vaccines have been developed to improve cancer immunotherapy in numerous clinical trials. Targeted delivery of antigens and adjuvants to DCs in vivo represents an important approach for the development of DC vaccines. However, nonspecific activation of systemic DCs and the preparation of optimal immunodominant tumor antigens still represent major challenges.

Methods: We loaded the immunogenic cell death (ICD) inducers human neutrophil elastase (ELANE) and Hiltonol (TLR3 agonist) into a-lactalbumin (a-LA)-engineered breast cancer-derived exosomes to form an in situ DC vaccine (HELA-EXOS). HELA-EXos were identified by transmission electron microscopy, nanoscale flow cytometry, and Western blot analysis. The targeting, killing, and immune activation effects of HELA-Exos were evaluated in vitro. The tumor suppressor and immune-activating effects of HELA-Exos were explored in immunocompetent mice and patientderived organoids.
\end{abstract}

Results: HELA-Exos possessed a profound ability to specifically induce ICD in breast cancer cells. Adequate exposure to tumor antigens and Hiltonol following HELA-EXO-induced ICD of cancer cells activated type one conventional DCs (CDC1s) in situ and cross-primed tumor-reactive $C D 8^{+} \mathrm{T}$ cell responses, leading to potent tumor inhibition in a poorly immunogenic triple negative breast cancer (TNBC) mouse xenograft model and patient-derived tumor organoids.

Conclusions: HELA-Exos exhibit potent antitumor activity in both a mouse model and human breast cancer organoids by promoting the activation of $\mathrm{CDC} 1 \mathrm{~s}$ in situ and thus improving the subsequent tumor-reactive $\mathrm{CD} 8^{+} \mathrm{T}$ cell responses. The strategy proposed here is promising for generating an in situ DC-primed vaccine and can be extended to various types of cancers.

Keywords: Breast cancer, Tumor-derived exosomes, Immunogenic cell death, Hiltonol, Type 1 conventional dendritic cells, DC vaccine

\footnotetext{
*Correspondence: Chunhuii.yuen@whu.edu.cn; wfb20042002@sina.com

${ }^{\dagger}$ Lanxiang Huang and Yuan Rong contributed equally to this work.

${ }^{1}$ Department of Laboratory Medicine, Zhongnan Hospital of Wuhan

University, No.169 Donghu Road, Wuhan, Wuchang District, China

7 Department of Laboratory Medicine, Wuhan Children's Hospital, Tongji

Medical College, Huazhong University of Science and Technology,

Wuhan, China

Full list of author information is available at the end of the article
}

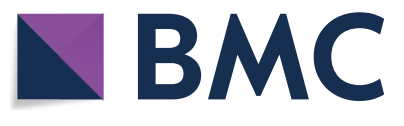

(c) The Author(s) 2022. Open Access This article is licensed under a Creative Commons Attribution 4.0 International License, which permits use, sharing, adaptation, distribution and reproduction in any medium or format, as long as you give appropriate credit to the original author(s) and the source, provide a link to the Creative Commons licence, and indicate if changes were made. The images or other third party material in this article are included in the article's Creative Commons licence, unless indicated otherwise in a credit line to the material. If material is not included in the article's Creative Commons licence and your intended use is not permitted by statutory regulation or exceeds the permitted use, you will need to obtain permission directly from the copyright holder. To view a copy of this licence, visit http://creativecommons.org/licenses/by/4.0/. The Creative Commons Public Domain Dedication waiver (http://creativeco mmons.org/publicdomain/zero/1.0/) applies to the data made available in this article, unless otherwise stated in a credit line to the data. 


\section{Graphic Abstract}

Scheme 1. Schematic illustration of HELA-Exos as an in situ DC-primed vaccine for breast cancer. (A) Allogenic breast cancer-derived exosomes isolated from MDA-MB-231 cells were genetically engineered to overexpress $a-L A$ and simultaneously loaded with the ICD inducers ELANE and Hiltonol (TLR3 agonist) to generate HELA-Exos. (B) Mechanism by which HELA-Exos activate DCs in situ in a mouse xenograft model ofTNBC. HELA-Exos specifically homed to the TME and induced ICD in cancer cells, which resulted in the increased release of tumor antigens, Hiltonol, and DAMPs, as well as the uptake of dying tumor cells by CDC1s. The activated $\mathrm{CDC} 1 \mathrm{~s}$ then cross-primed tumor-reactive $\mathrm{CD} 8+\mathrm{T}$ cell responses. (C) HELA-Exos activated DCs in situ in the breast cancer patient PBMC-autologous tumor organoid coculture system. Abbreviations: DCs: dendritic cells; a-LA: a-lactalbumin; HELA-Exos: Hiltonol-ELANE-a-LA-engineered exosomes; ICD: immunogenic cell death; ELANE: human neutrophil elastase; TLR3: Toll-like receptor 3; TNBC: triple-negative breast cancer; TME: tumor microenvironment; DAMPs: damage-associated molecular patterns; CDC1s: type 1 conventional dendritic cells; PBMCs: peripheral blood mononuclear cells

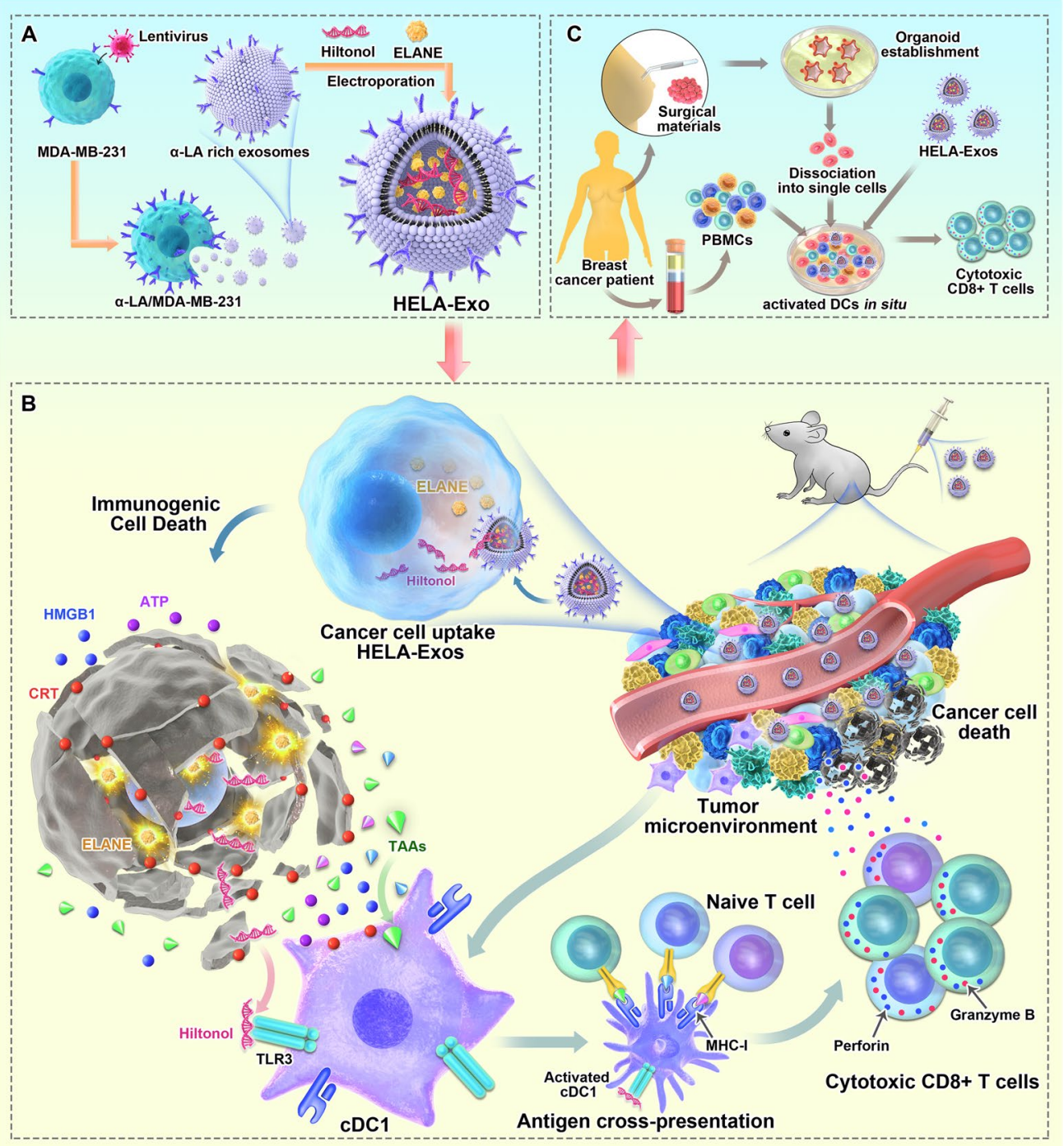




\section{Introduction}

As the most potent antigen-presenting cells (APCs), dendritic cells (DCs) are central to the initiation and regulation of innate and adaptive immunity in the tumor microenvironment (TME), with the ability to present tumor-associated antigens (TAAs) on MHC molecules and provide costimulatory/soluble factors to shape antitumor $\mathrm{T}$ cell responses [1]. As such, many kinds of DC-targeted vaccines have been developed to improve cancer immunotherapy in numerous clinical trials $[2,3]$. Targeted delivery of antigens and adjuvants to DCs in vivo represents an important approach to enhance the antitumor effects of DCs [4]. In contrast to ex vivo generation, in vivo activation and mobilization of DCs allows vaccines to be produced on a larger scale and allows direct activation of natural DC subsets at multiple sites in vivo [5]. However, because DC-target receptors, such as DEC205 [6] and DC-SIGN [7], are not exclusively expressed on tumor-infiltrating DCs, this strategy can also induce antigen-nonspecific activation of the immune system and potential side effects in the clinic $[8,9]$. In addition, the other major challenge for the implementation this strategy is the identification of optimal immunodominant tumor antigens, such as TAA, TSA and even patient-specific neoantigens [10]. Efforts to address these concerns are critical for making DC vaccines clinically feasible.

The type of cell death induced in tumor cells can influence their efficacy in inducing immunity [11]. Immunogenic cell death (ICD) enhances tumor antigen exposure, boosts the release of immune-stimulating tumor cell content, and favors the uptake of dying tumor cells by DCs [12-14]. In this context, ICD has been approved for whole-tumor lysate preparations for autologous DC vaccines [15]. Thus, induction of ICD in tumor cells would be an effective way to maintain adequate exposure of DCs to tumor antigens and bypass the need for identification of optimal immunodominant antigens. On the other hand, DCs usually exhibit poor maturation in the TME and are thus less effective in presenting tumor antigens [16]. Adjuvants that drive immunogenic DC activation, particularly agonists of TLR3, are being actively investigated [6]. Administration of TLR3 agonists is extraordinarily effective in inducing maturation of type one conventional DCs (cDC1s) $[17,18]$, which are necessary for inducing cellular immunity and are linked to increased survival in patients with certain cancer types due to their efficient processing and cross-presentation of exogenous antigens on MHC class I molecules to activate CD8 ${ }^{+} \mathrm{T}$ cells and prime $\mathrm{T}_{\mathrm{H}} 1$ cell responses [19-22]. Thus, targeted delivery of ICD inducers and TLR3 agonists into tumor cells would be an ideal way to activate tumor-infiltrating DCs in situ and avoid interactions in the immune milieu of other sites.

In recent years, exosomes have come into focus as "natural nanoparticles" for use as drug delivery vehicles owing to their low cytotoxicity, ability to maximize the bioavailability of drugs, and exquisite target-homing specificity $[23,24]$. The therapeutic potential of exosomes is reflected by their application in an increasing number of clinical trials (ClinicalTrials.gov) for the treatment of chronic kidney diseases [25], non-smallcell lung cancer [26], colon cancer [27], type 1 diabetes, and severe COVID-19 [28]. Notably, the chemotherapeutic doxorubicin (Dox) was more stable when loaded in breast cancer-derived exosomes and accumulated more efficiently in tumors than free Dox for the treatment of breast cancer in a mouse model [29]. Therefore, tumor-derived exosomes (Texs) can be used as a cell-free therapeutic carrier to infiltrate the TME for in situ DC activation.

Herein, we developed and optimized a formulation of target-specific exosomes loaded with Hiltonol (TLR3 agonist) and the ICD inducer human neutrophil elastase (ELANE) [30] to form an in situ DC vaccine for breast cancer treatment. $\alpha$-Lactalbumin $(\alpha-\mathrm{LA})$, a breast-specific immunodominant protein expressed in the majority of human breast cancers [31], was further genetically enriched on the surface of exosomes as a specific tumor-homing protein to enhance targeting capability and immunogenicity [32]. In both a mouse model and patient-derived tumor organoids, these Hiltonol-ELANE- $\alpha$-LA-engineered exosomes (HELAExos) possessed a profound ability to accumulate in cancer cells after systemic administration and exerted improved therapeutic effects compared to those of free Hiltonol (Scheme 1). Therefore, the combination of TLR3 agonists with ICD inducers based on cell-free exosomes offers a powerful and novel therapeutic platform for designing DC vaccines for breast cancer.

\section{Results}

\section{Characterization and optimization of HELA-Exos}

To determine whether $\alpha$-LA is a specific dominant epitope in breast cancer, we first evaluated the expression of $\alpha$-LA in different cell lines. As predicted, the triplenegative breast cancer (TNBC) cell line MDA-MB-231 and the non-TNBC cell lines MCF7, MDA-MB-435S, and SKBR3 expressed significantly higher $\alpha$-LA mRNA levels than other cell lines, including the nontransformed human mammary epithelial cell line MCF10A and other types of cancer cells (Fig. 1A). TNBC represents a class of poorly immunogenic tumors associated with low survival rates and high resistance to current immunotherapies $[33,34]$. Therefore, MDA-MB-231 cells were genetically 


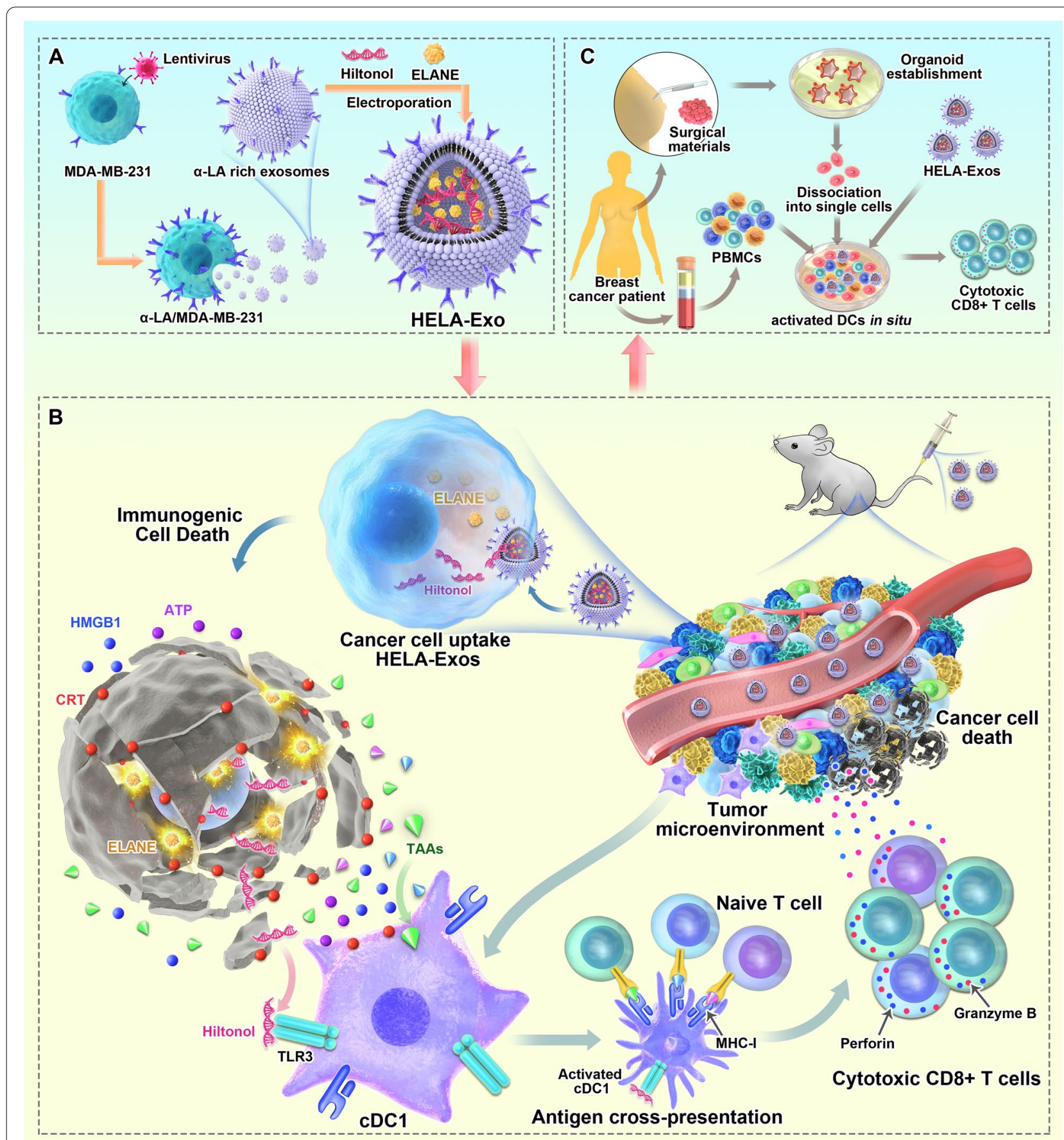

Scheme 1 Schematic illustration of HELA-Exos as an in situ DC-primed vaccine for breast cancer. A Allogenic breast cancer-derived exosomes isolated from MDA-MB-231 cells were genetically engineered to overexpress a-LA and simultaneously loaded with the ICD inducers ELANE and Hiltonol (TLR3 agonist) to generate HELA-EXos. B Mechanism by which HELA-Exos activate DCs in situ in a mouse xenograft model ofTNBC. HELA-EXos specifically homed to the TME and induced ICD in cancer cells, which resulted in the increased release of tumor antigens, Hiltonol, and DAMPs, as well as the uptake of dying tumor cells by $\mathrm{CDC} 1 \mathrm{~s}$. The activated $\mathrm{CDC} 1 \mathrm{~s}$ then cross-primed tumor-reactive $\mathrm{CD} 8^{+} \mathrm{T}$ cell responses. (C) HELA-Exos activated DCs in situ in the breast cancer patient PBMC-autologous tumor organoid coculture system. Abbreviations: DCs: dendritic cells; a-LA: a-lactalbumin; HELA-Exos: Hiltonol-ELANE-a-LA-engineered exosomes; ICD: immunogenic cell death; ELANE: human neutrophil elastase; TLR3: Toll-like receptor 3; TNBC: triple-negative breast cancer; TME: tumor microenvironment; DAMPs: damage-associated molecular patterns; CDC1s: type 1 conventional dendritic cells; PBMCs: peripheral blood mononuclear cells 


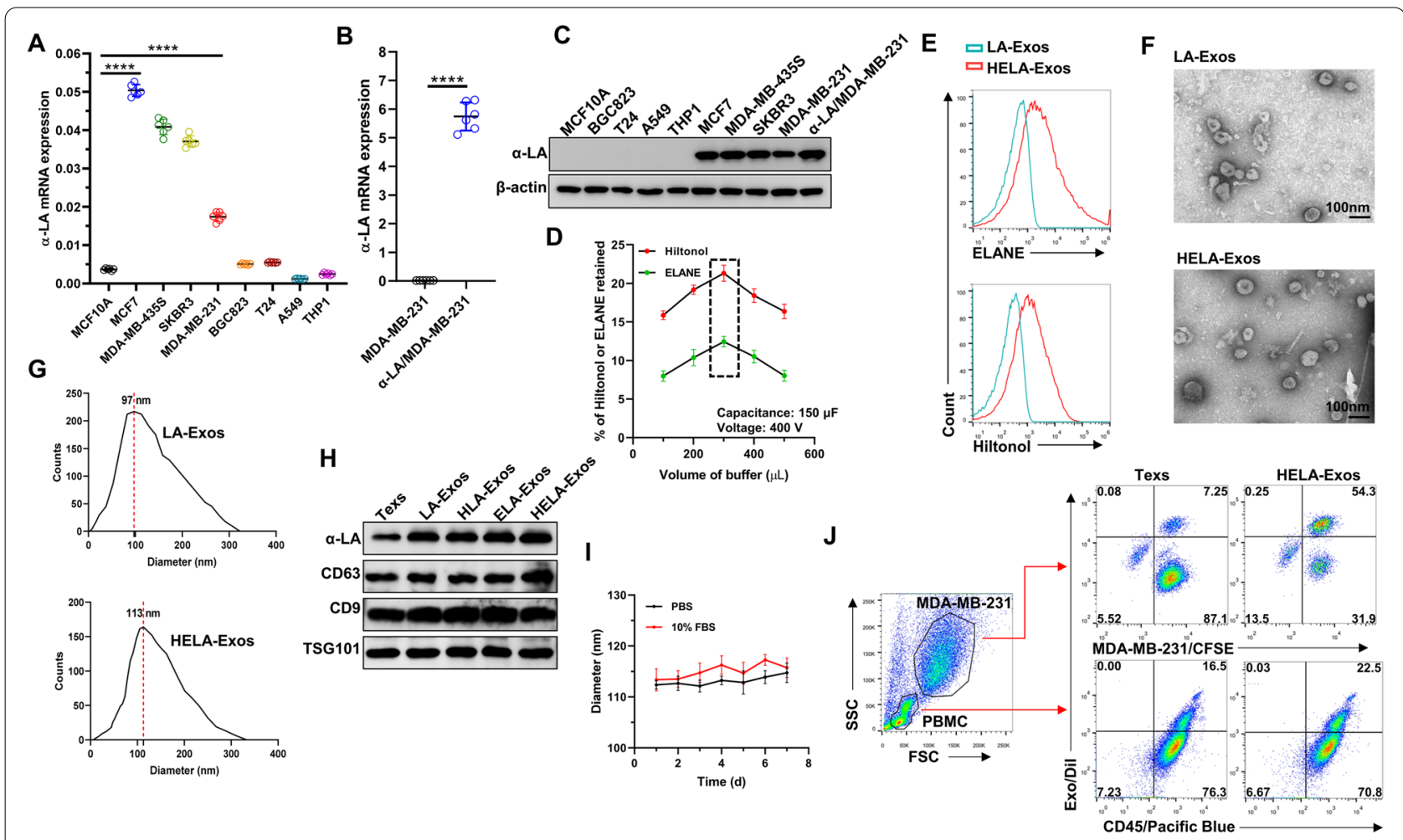

Fig. 1 Preparation, characterization, and targeting of HELA-EXOs. A and B Relative abundance of a-LA mRNA in different cell lines (the human normal breast epithelial cell line MCF-10A, breast adenocarcinoma cell line MCF7, breast cancer cell line MDA-MB-435S, breast cancer cell line SKBR3, TNBC cell line MDA-MB-231, gastric adenocarcinoma cell line BGC-823, transitional cell carcinoma cell line T24, alveolar basal epithelial adenocarcinoma cell line A549, and leukemic cell line THP-1) and infected MDA-MB-231 cells by GPCR; data were normalized to GAPDH. The data are presented as the mean $\pm S D ; n=6$. A t test was performed for statistical analysis (***: $P<0.0001$ ). C Western blot analysis for detecting the expression of a-LA protein in cells as in A. $\beta$-Actin was used as a loading control. D FITC-labeled ELANE and CX-Rhodamine-labeled Hiltonol were electroporated with exosomes at $400 \mathrm{~V} 150 \mu \mathrm{F}$ in the volume of buffer shown on the $\mathrm{x}$-axis, and ELANE and Hiltonol loading were determined based on the fluorescent standard curve. The data are presented as the mean $\pm S D ; n=3$. E The loading of ELANE and Hiltonol in exosomes was characterized by nanoscale flow cytometry. F Transmission electron micrograph of LA-Exos and HELA-Exos. Scale bar, $100 \mathrm{~nm}$. G Size distribution of LA-Exos and HELA-Exos measured by a particle sizer. $\mathbf{H}$ Western blot analysis for detecting the expression of the a-LA protein and exosomal markers in Texs, LA-EXos, HLA-EXos, ELA-EXos, and HELA-EXos. I Diameter change of HELA-Exos from day 1 to day 7. The data are presented as the mean \pm SD; $n=3$. J Flow cytometry analysis of the cellular uptake of MDA-MB-231 cells or PBMCs after incubation with Texs-Dil or HELA-Exos-Dil for $2 \mathrm{~h}$ in the coculture model

engineered to overexpress $\alpha$-LA (Fig. $1 B$ and C), and exosomes derived from MDA-MB-231 cells (Texs) and $\alpha$-LA-overexpressed cells (LA-Exos) were enriched by ultracentrifugation. Hiltonol and ELANE were then loaded into LA-Exos via electroporation, and the optimal loading conditions were $400 \mathrm{~V}$ and $150 \mu \mathrm{F}$ in $300 \mu \mathrm{L}$ of buffer, as determined by measuring the fluorescence intensity of FITC-labeled ELANE and CX-rhodaminelabeled Hiltonol (Fig. S1A-1D, Fig. 1D) and nanoscale flow cytometry (Fig. 1E). Finally, the constructed HELAExos showed a slightly larger average diameter than the parental LA-Exos (Fig. 1F and G), exhibited increased $\alpha$-LA expression (Fig. $1 \mathrm{H}$ ), and were well dispersed in phosphate-buffered saline (PBS, $\mathrm{pH} 7.4$ ) and 10\% fetal bovine serum (FBS), with long-term stability (Fig. 1I). Thus, these results suggest that HELA-Exos may have the potential to be used in in vitro and in vivo studies.
In vitro targeted delivery and killing capacity of HELA-Exos

To evaluate the targeting specificity of HELA-Exos, CFSE-labeled MDA-MB-231 cells were mixed with human peripheral blood mononuclear cells (PBMCs) at a 5:1 ratio, and then DiI-labeled HELA-Exos were added to this cell mixture. Compared with Texs, HELA-Exos showed a marked enrichment in MDA-MB-231 cells $(53.81 \% \pm 2.95 \%$ vs. $8.12 \% \pm 2.44 \%)$, while there was no difference in PBMCs (Fig. 1J) and lung cancer A549 cells (Fig. S1E). These results indicate that HELA-Exos have excellent targeting specificity for breast cancer cells.

ELANE selectively kills a broad range of cancer cells and exhibits minimal toxicity to noncancer cells [30]. In this study, ELANE-loaded Exos (HELA-Exos and ELA-Exos) effectively killed MDA-MB-231 cells, as determined by quantifying the calcein-AM fluorescence signal (Fig. 2A and B), PI staining, and PARP1 


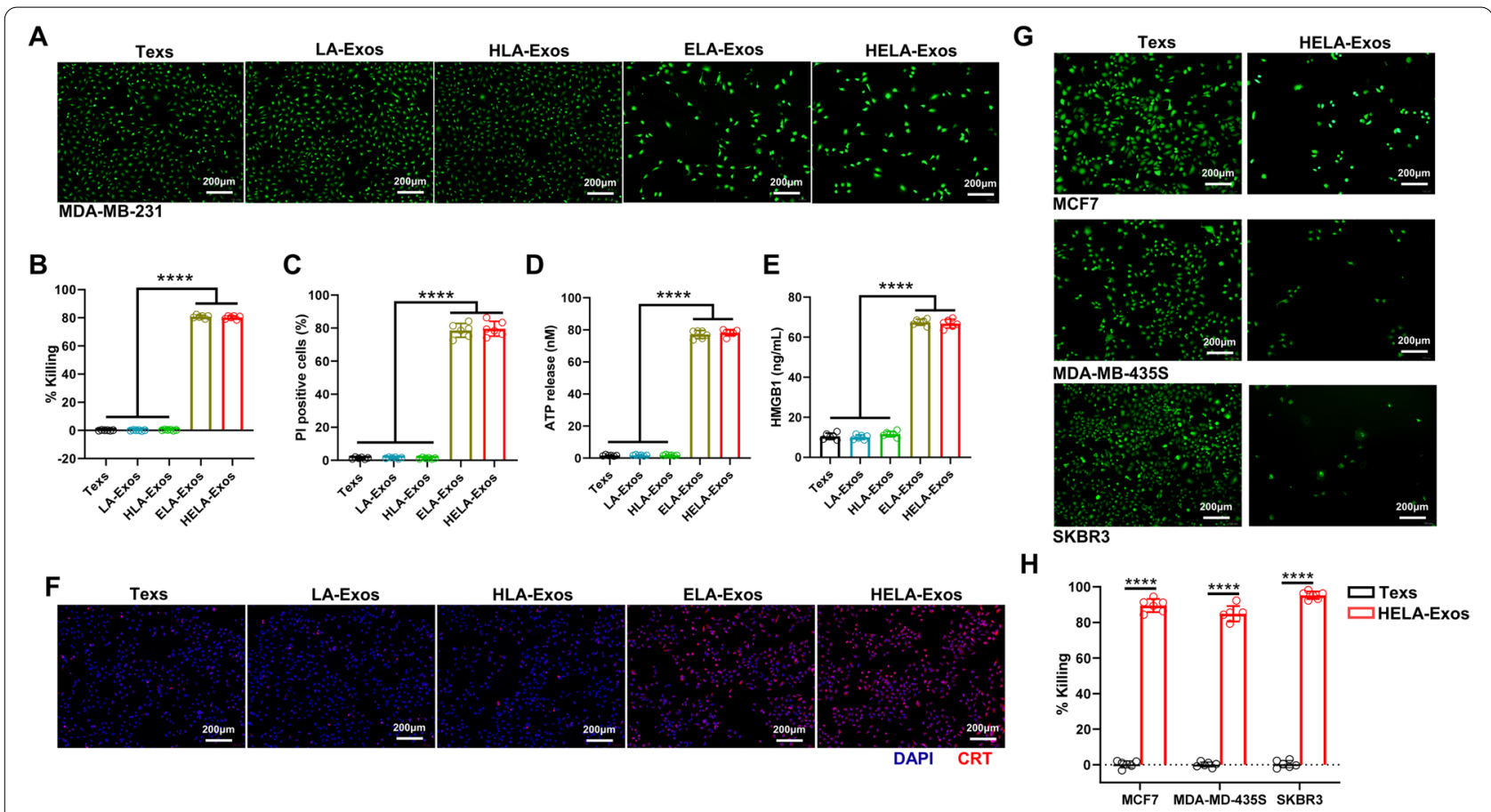

Fig. 2 In vitro killing capacity of HELA-EXos. A to F MDA-MB-231 cells were treated with Texs, LA-Exos, HLA-Exos, ELA-Exos, or HELA-Exos for 48 h. A and $\mathbf{B}$ Representative images of calcein-AM fluorescence and the quantitative detection of MDA-MB-231 cell viability. Scale bar, $200 \mu \mathrm{m}$. The data are presented as the mean $\pm S D ; n=6$. One-way ANOVA was performed for statistical analysis $(* * *: P<0.0001)$. C Apoptosis analysis by PI staining and flow cytometry. The data are presented as the mean $\pm S D ; n=6$. One-way ANOVA was performed for statistical analysis $(* * * *: P<0.0001)$. D and E ATP release and HMGB1 release were evaluated by ELISA. The data are presented as the mean $\pm S D ; n=6$. One-way ANOVA was performed for statistical analysis (***: P<0.0001). F The surface exposure of CRT on MDA-MB-231 cells was evaluated by CLSM. Blue: DAPI; Red: CRT. Scale bar, $200 \mu \mathrm{m}$. G and H MCF7, MDA-MB-435S, and SKBR3 cells were treated with Texs or HELA-Exos for $48 \mathrm{~h}$. Representative images of calcein-AM fluorescence and the quantitative detection of cell viability in different cells. Scale bar, $200 \mu \mathrm{m}$. The data are presented as the mean $\pm S D ; n=6$. At test was performed for statistical analysis (****: $P<0.0001$ ). ICD: immunogenic cell death; CRT: calreticulin; CLSM: confocal laser scanning microscopy

and CASP3 expression (Fig. 2C, Fig. S2A and B). Concurrently, the levels of three hallmarks of ICD, namely, calreticulin (CRT), ATP and HMGB1, were significantly increased in HELA-Exo-treated cells compared with parental Exo-treated cells (Fig. 2D-F). These results clearly indicate that HELA-Exos induce potent ICD in breast cancer cells in vitro. To assess the general applicability of HELA-Exos in other subtypes of breast cancer cells, MCF7, MDA-MB-435S, and SKBR-3 cells were further treated with HELA-Exos. The results of the calcein-AM fluorescence quantitative test showed that the HELA-Exos more effectively killed MCF7, MDA-MB-435S, and SKBR3 cells compared with the parental Texs (Fig. 2G and H). These results demonstrate that HELA-Exos exhibit general killing capacity in different types of breast cancer cells.

\section{HELA-Exos stimulate the antigen cross-presentation activity of $\mathrm{CDC1s}$ and induce potent $\mathrm{CD8}{ }^{+} \mathrm{T}$ cell responses in vitro}

Next, we further verified whether HELA-Exos kill normal cells before evaluating their immune activation ability. Human normal cells (PBMCs, PBMC-derived DCs, and MCF10A cells) were treated with HELAExos, and the results confirmed that HELA-Exos were not toxic to all human normal cells tested (Fig. 3A and B). Then, the DC-priming potential of HELA-Exos was evaluated in a cancer cell-DC-CD8 ${ }^{+} \mathrm{T}$ cell coculture model (Fig. 3C). Flow cytometry analysis revealed that HELA-Exo treatment induced significantly higher fluorescence intensity of CD141 (a marker of $\mathrm{CDC} 1 \mathrm{~s}$ ) and HLA-A2 in DCs than Hiltonol, while CD1c (a marker of cDC2s) and HLA-DR showed no obvious difference (Fig. 3D). These results indicate that HELA-Exos are more potent in inducing maturation of the $\mathrm{CDC} 1 \mathrm{sub}-$ set than Hiltonol. $\mathrm{cDC} 1 \mathrm{~s}$ are efficient in the processing and cross-presentation of TAAs on MHC-I molecules to activate $\mathrm{CD}^{+} \mathrm{T}$ cells in breast cancer [35]. Indeed, compared to Hiltonol treatment, HELA-Exo treatment had a more robust priming effect on $\mathrm{CD}^{+} \mathrm{T}$ cells, as evidenced by higherproduction of granzyme $\mathrm{B}$ and perforin, increased expression of CD69 (activation marker) and lower expression of PD1 in $\mathrm{CD}^{+} \mathrm{T}$ cells (Fig. 3E and $\mathrm{F}$ ), which induce specific cytotoxic activity against 


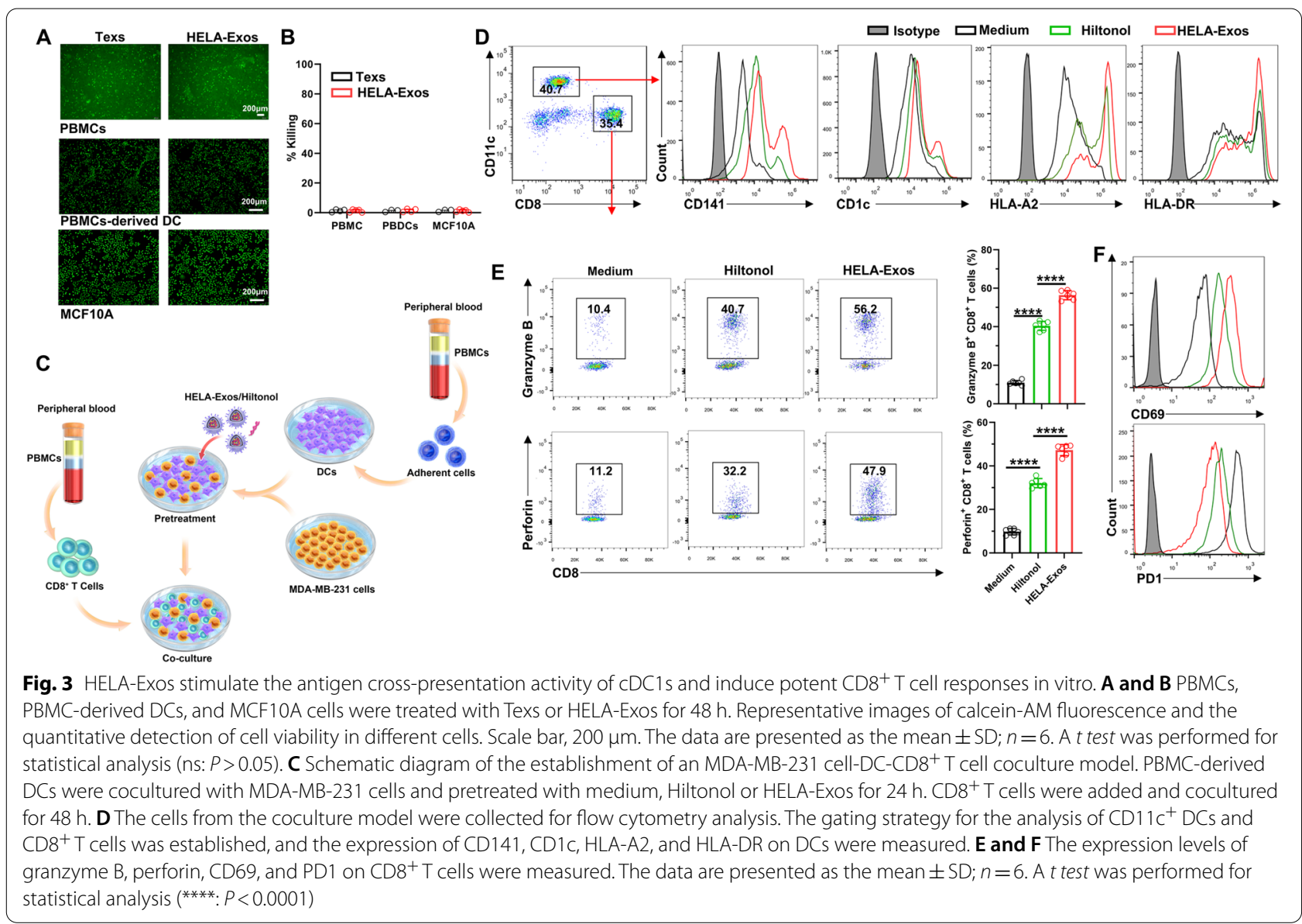

breast cells, rather than lung cancer cells or normal breast cells in vitro (Fig. S2C). Overall, these results demonstrate that the HELA-Exos selectively induce ICD in breast cancer cells and thus contribute to $\mathrm{CDC} 1$ maturation and $\mathrm{CD} 8^{+} \mathrm{T}$ cell activation in vitro.

\section{Antitumor efficacy of HELA-Exos in mice with orthotopic TNBC in vivo}

We next investigated the biodistribution and tumor accumulation of HELA-Exos in vivo. CX-Rhodamine-labeled Hiltonol and HELA-Exos-DiI were administered intravenously to orthotopic tumor-bearing mice. Compared with the free CX-Rhodamine-labeled Hiltonol, a stronger fluorescence signal was detected in the tumor region of HELA-Exos-DiI-treated mice at $2 \mathrm{~h}$ post-injection, and it persisted to $24 \mathrm{~h}$ post-injection (Fig. S3A), implying an excellent targeting ability of HELA-Exos. The in vivo pharmacokinetic analysis revealed that the half-life of HELA-Exos showed no obvious changes between the first and last treatment, and the HELA-Exos exhibited superior retention in the blood compared to that of Hiltonol $(8.41 \pm 0.37 \mathrm{~h}$ vs. $3.71 \pm 0.90 \mathrm{~h}$, Fig. S3B), which demonstrates the stability of HELA-Exos in vivo.
Encouraged by biodistribution results, the tumor inhibition of HELA-Exos was next evaluated. The therapeutic potential of the HELA-Exos was investigated after their systemic administration in an orthotopic TNBC mouse model according to the treatment regimen presented in Fig. 4A. MDA-MB-231/luc cells were inoculated into orthotopic mammary fat and allowed to grow for approximately 21 days to $\sim 100 \mathrm{~mm}^{3}$ before administration of Hiltonol or HELA-Exos. Tumor growth was recorded by measuring the bioluminescence signal from luciferasetagged MDA-MB-231 cancer cells (Fig. 4B). The intensity of the bioluminescence signal and tumor volume were found drastically decrease over the duration of treatment in the groups treated with Hiltonol or HELA-Exos compared with the medium control group, and more importantly, the group that received HELA-Exos had the lowest bioluminescent signal intensity and tumor volume (Fig. 4C and D). The therapeutic efficacy of the HELAExos was further evidenced by the fact that compared to breast tissues in the medium control and Hiltonol-treated groups, isolated breast cancer tissues exhibited the lowest Ki67 expression and the highest CASP3 and CRT expression (Fig. 4E). Thus, it was evident that HELA-Exos 


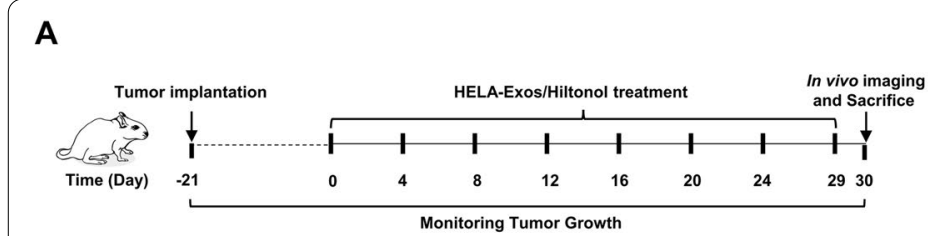

B

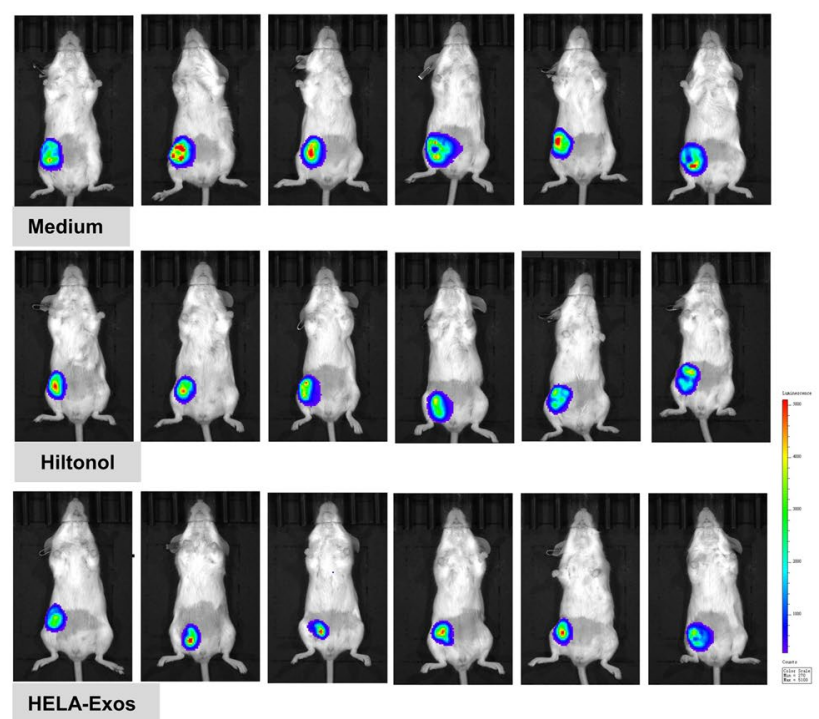

C

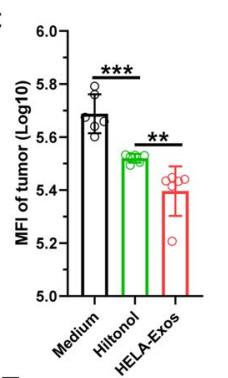

D

$\mathrm{E}$
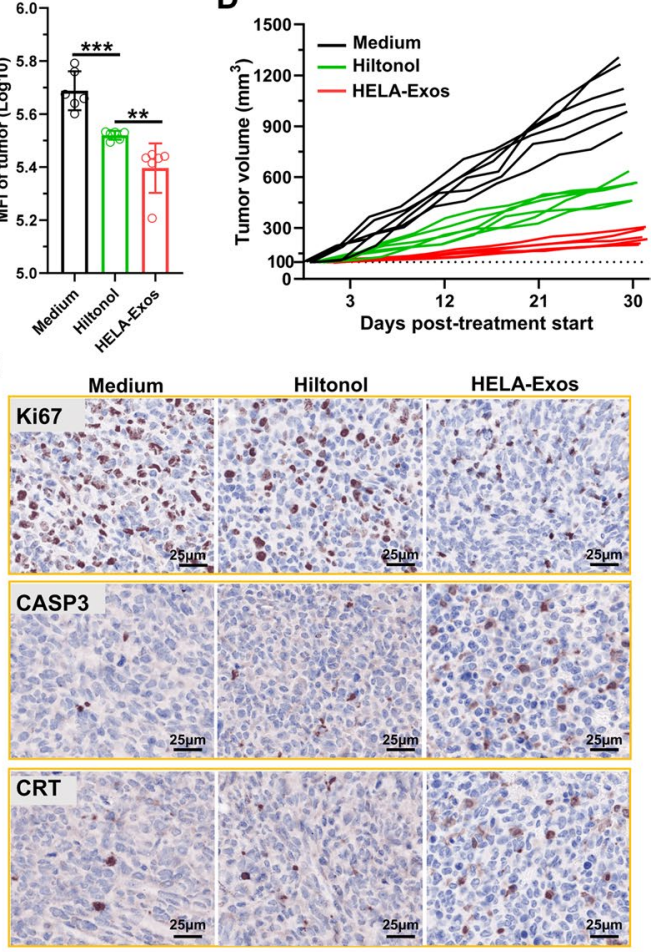

Fig. 4 Antitumor efficacy of HELA-Exos in an orthotopic TNBC model in vivo. Establishment of a xenograft human tumor model in immunocompetent mice, as described in the methods section, and initiation of therapy. A Schematic diagram of the vaccine dosing regimen in Balb/c mice with orthotopic breast cancer. B and C In vivo tumor imaging in mice was performed using an IVIS Spectrum In Vivo Imaging System at Day 30 after initiation of therapy, and the bioluminescence intensity of the tumor was proportional to the size. The data are presented as the mean $\pm \mathrm{SD} ; n=6$. A t test was performed for statistical analysis (**: $P<0.001 ;{ }^{* *}: P<0.01$ ). D Tumor size was measured every other day after treatment initiation. Tumor growth curves for each mouse are shown. $\mathbf{E}$ Immunohistochemical staining examination of Ki67, CASP3, and CRT in tumor tissue. Scale bar, 25 Mm. MFI: mean fluorescence intensity

exhibited higher therapeutic efficacy for breast cancer than free Hiltonol. Histological examination of the major organs and biochemical analysis revealed that systemic administration of HELA-Exos did not cause severe side effects in mice (Fig. S3C and S3D). Overall, these results demonstrated that HELA-Exos possess promising therapeutic potential for breast cancer.

\section{HELA-Exos induce intratumoral accumulation of $\mathrm{CDC} 1 \mathrm{~s}$ and $\mathrm{CD}^{+} \mathrm{T}$ cells}

Immune cells in isolated breast cancer tissues were further analyzed to evaluate the immune activation ability of HELA-Exos in vivo. Immunofluorescence results demonstrated that HELA-Exo treatment markedly increased the infiltration of DCs, especially the $\mathrm{CDC1}$ subset of mice $\left(\mathrm{CD} 11 \mathrm{c}^{+} \mathrm{CD} 103^{+}\right)$, in both the TME and draining lymph nodes, while the infiltration of macrophages was basically unchanged compared to that observed during Hiltonol treatment (Fig. S4, Fig. 5A). Flow cytometry analysis also confirmed the potent accumulation and maturation of the $\mathrm{CDC} 1$ subset in the TME after HELA-Exo treatment (Fig. 5B), suggesting that the targeted codelivery of an ICD inducer with a TLR3 agonist efficiently primed DCs in situ. In addition, compared to the medium control or Hiltonol treatment, HELA-Exo treatment markedly increased infiltration (Fig. 5C), granzyme B and perforin production (Fig. 5D and E), and CD69 expression and decreased PD1 expression (Fig. 5F) in specific cytotoxic $\mathrm{CD}^{+} \mathrm{T}$ cells within the TME (Fig. S5). In addition, despite demonstrating immune activation ability, repeated injections of HELA-Exos induced greatly reduced systemic inflammation relative to that induced by free Hiltonol (Fig. S6). Notably, depletion of DCs (Fig. 6A and B, Fig. S7A) significantly blocked the antitumor effects of HELA-Exos in breast cancer (Fig. 6C-E), as well as its active effects on $\mathrm{CD} 8^{+} \mathrm{T}$ cells (Fig. $6 \mathrm{~F}-\mathrm{H}$, Fig. S7B) within the TME. Taken together, these results demonstrate that HELA-Exos can effectively promote the infiltration and maturation of the $\mathrm{CDC} 1$ subset in situ and boost antitumor $\mathrm{CD} 8^{+} \mathrm{T}$ cell immunity within the TME. 

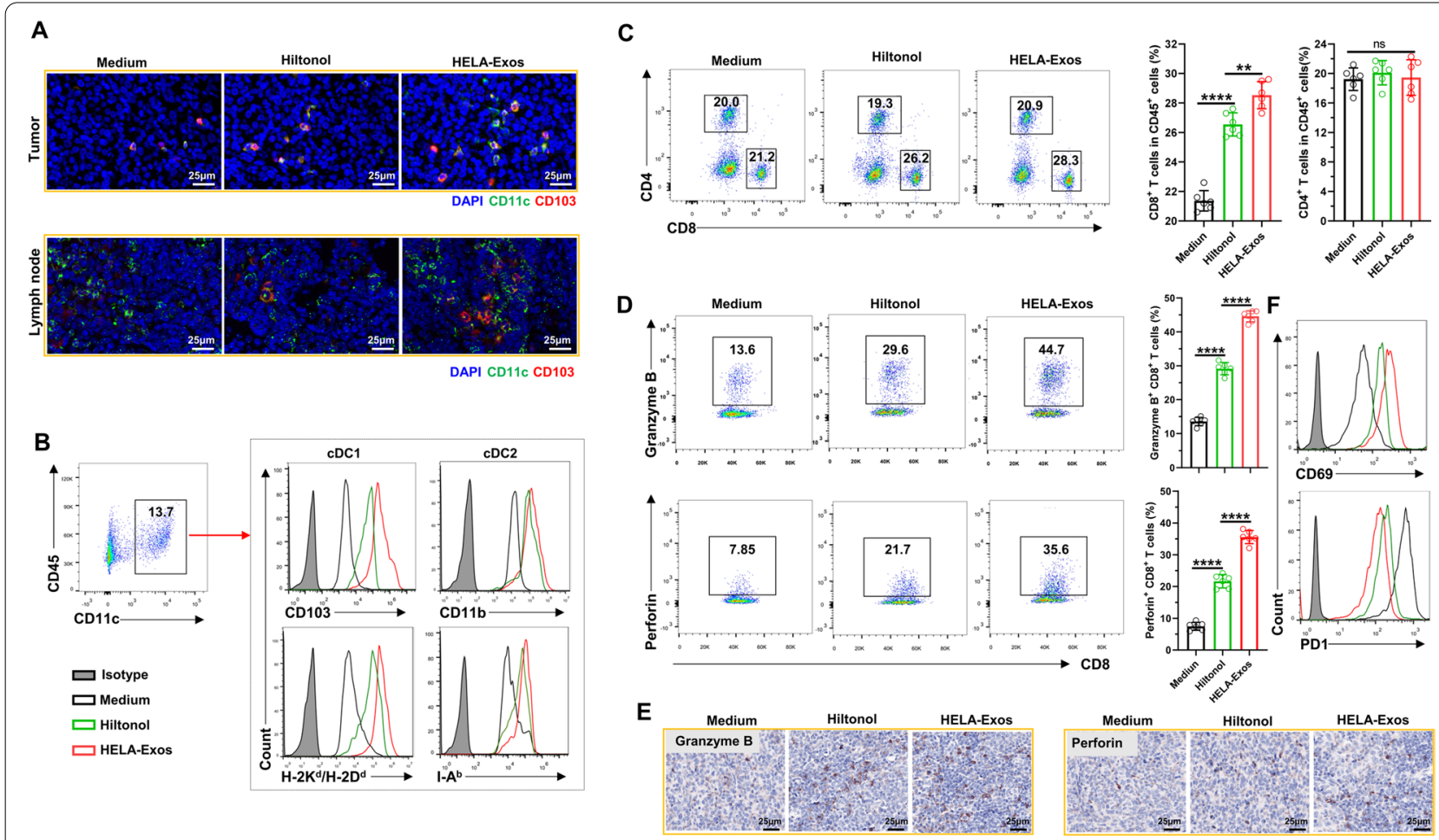

Fig. 5 HELA-EXOs induce intratumoral accumulation of $C D C 1 s$ and $C D 8+T$ cells. Balb/c mice with orthotopic breast cancer were sacrificed at day 30 after treatment initiation. Tumor tissues and draining lymph node immune infiltrates were analyzed by IF, flow cytometry and IHC. A Tumor tissue- and draining lymph node-infiltrating CDC1s were detected by IF. Blue: DAPI; Green: CD11 c; Red: CD103. Scale bar, $25 \mu \mathrm{m}$. B Gating strategy for the analysis of CD11 $\mathrm{C}^{+} \mathrm{DC}$; the expression of CD103, CD1 1 b, H-2K $/ \mathrm{H}-2 \mathrm{D}^{\mathrm{d}}(\mathrm{MHC}-\mathrm{I})$, and I-A $\mathrm{A}$ (MHC-II) on DCs was measured using flow cytometry. $\mathbf{C}$ The percentages of tumor-infiltrating $\mathrm{CD}^{+}$or $\mathrm{CD} 8^{+} \mathrm{T}$ cells were measured using flow cytometry. $\mathbf{D}$ to $\mathbf{F}$ Tumor-infiltrating $\mathrm{CD} 8^{+} \mathrm{T}$ cells were analyzed for the expression of the cytotoxicity markers granzyme B and perforin, the activation marker CD69 and the immunosuppressive marker PD1. The data are presented as the mean $\pm \mathrm{SD} ; n=6$. $t$ test and one-way ANOVA were performed for statistical analysis $\left(* * * *: P<0.0001 ;{ }^{* *}\right.$ : $P<0.01 ;$ ns: $P>0.05)$. Scale bar, $25 \mu \mathrm{m}$. IF: immunofluorescence; IHC: immunohistochemistry

\section{HELA-Exos profoundly enhance CDC1 antigen cross-presentation and tumor-reactive $\mathrm{CD}^{+} \mathrm{T}$ cell generation in a patient-derived tumor organoid coculture system}

To confirm the therapeutic potential of this strategy for human cancer therapy, we then tested whether HELAExos stimulate human $\mathrm{T}$ cells in patient-derived tumor organoids (PDOs). According to a previously reported protocol [36], PBMCs from breast cancer patients were cocultured with autologous PDOs for 2 weeks. In addition, Hiltonol or HELA-Exos were administered to the organoid-PBMC coculture systems (Fig. S8). Flow cytometry and immunofluorescence analysis revealed that the infiltration of CD $141^{+} \mathrm{cDC} 1 \mathrm{~s}$ and $\mathrm{CD} 8^{+} \mathrm{T}$ cells (Fig. 7A$\mathrm{C}$ ), as well as the production of granzyme $\mathrm{B}$ and perforin in $\mathrm{CD}^{+} \mathrm{T}$ cells (Fig. 7D), were significantly increased in HELA-Exo-treated PDOs compared to the Hiltonolor medium-treated groups. These results suggest that HELA-Exos are effective in enhancing DC activation and the subsequent potent priming of tumor-reactive $C D 8^{+}$ $\mathrm{T}$ cells in autologous PDOs.
The growth-inhibitory effects of HELA-Exos in autologous PDOs were further evaluated by MTS assays and live-dead staining. Consistent with the aforementioned in vitro and in vivo observations, HELA-Exo treatment significantly inhibited organoid growth (Fig. 7E, Table S1) and induced potent cell death (Fig. 7F) in autologous PDOs. More importantly, HELA-Exos showed more potency than Hiltonol in autologous PDOs. Overall, these results suggest that HELA-Exos exhibit potent antitumor activity in breast cancer because they promote the activation of DCs in situ and subsequent tumor-reactive $\mathrm{CD}^{+} \mathrm{T}$ cell priming.

\section{Discussion}

TNBC, a specific subtype of breast cancer characterized by lack expression of estrogen receptor (ER), progesterone receptor (PR), and human epidermal growth factor receptor 2 (HER2), makes up approximately 15\% of all breast cancers [37]. Due to its special molecular phenotype, TNBC is not sensitive to endocrine therapy or molecular targeted therapy, and the efficacy of 


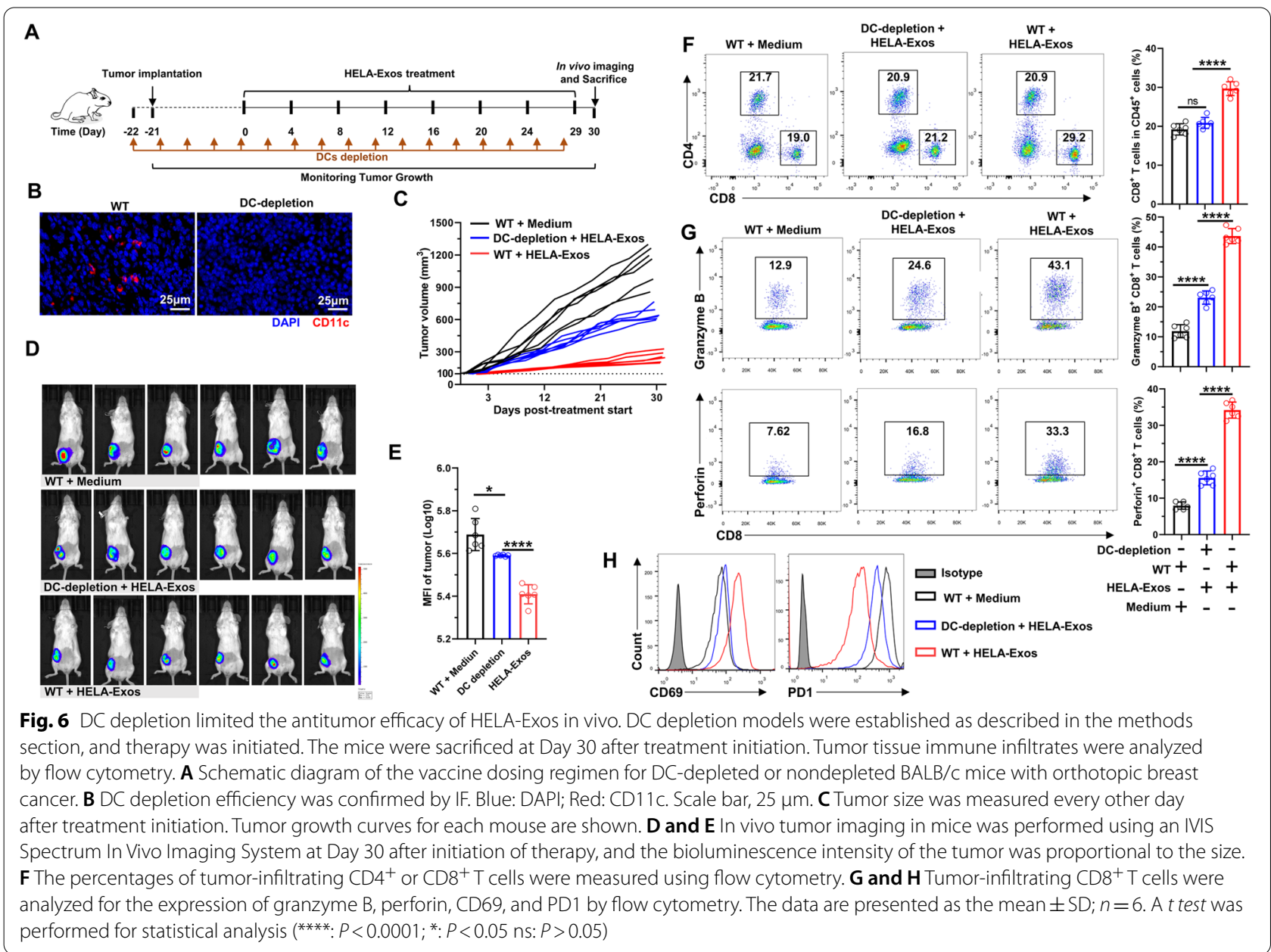

conventional postoperative adjuvant chemoradiotherapy is poor. The median survival time of TNBC after metastasis is only 13.3 months, and the recurrence rate after surgery is as high as $25 \%$ [38]. Therefore, the development of new TNBC treatment strategies has become an urgent clinical need. In the present study, we developed an in situ DC vaccine (HELA-Exos) by using engineered breast cancer-derived exosomes to codeliver the ICD inducers ELANE and Hiltonol for the treatment of breast cancer. HELA-Exos exhibit potent antitumor activity in both a mouse model and human organoids of breast cancer by promoting the activation of $\mathrm{CDC} 1 \mathrm{~s}$ in situ and thus improving the subsequent tumor-reactive $\mathrm{CD}^{+} \mathrm{T}$ cell responses.

To date, several vaccines targeting DCs are currently being tested for breast cancer in clinical trials. The DCtargeted vaccine oxidized mannan-MUC-1 dramatically decreased the recurrence rate in 31 patients with breast cancer $(12.5 \%$ vs. $60 \%$ in patients who received placebo) at 15 years of follow-up [39]. Although many of these vaccines are promising and have been shown to be successful in breast cancer, a large population of patients still do not benefit from these therapies [40]. The potential reasons may include two factors: 1 ) $\mathrm{cDC} 1 \mathrm{~s}$ usually exhibit poor maturation in the TME and are thus less effective in presenting tumor antigens [16], and 2) the intrinsic tumor antigen heterogeneity in the breast cancer TME may result in limited selective pressure from tumor-infiltrating cells [41]. Compared with free Hiltonol, the HELA-Exos investigated in this study, consisting of the TLR3 agonist Hiltonol, the ICD inducer ELANE and the immunodominant antigen $\alpha$-LA, effectively activated $\mathrm{CDCs}$ and improved antitumor $\mathrm{CD} 8^{+} \mathrm{T}$ cell responses. Based on these findings, HELA-Exos can potentially serve as a robust therapeutic regimen for breast cancer.

TLR3 stimulation is a potent strategy to induce the activation and maturation of $\mathrm{cDC} 1 \mathrm{~s}$ in the breast cancer TME $[42,43]$. cDC1s are critical for TME immunosurveillance and preferentially infiltrate TNBCs 


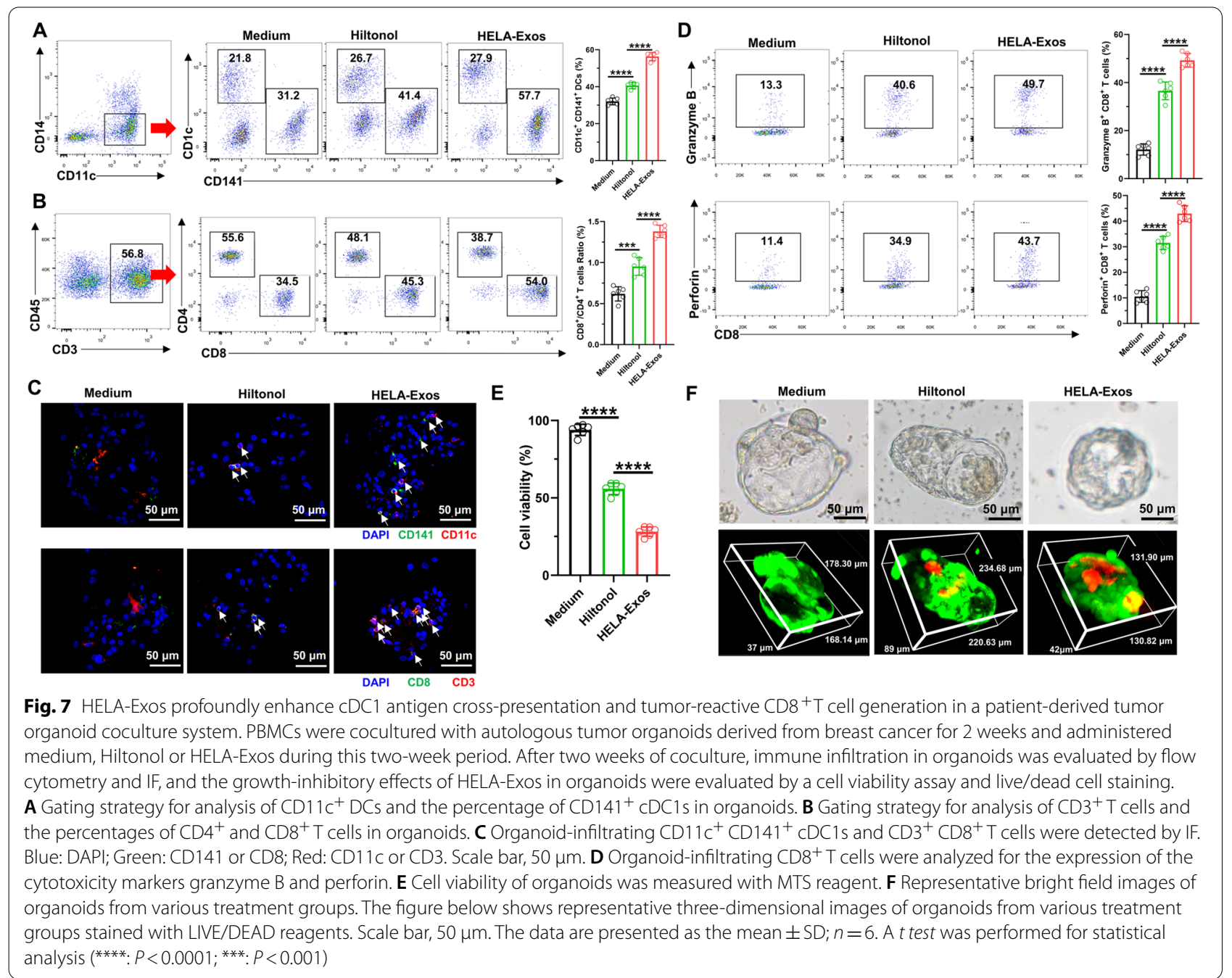

compared to the other two subtypes, and they predict good clinical outcomes in breast cancer [35, 42]. The FDA has granted the orphan drug designation to the TLR3 agonist poly(I:C)-expressing rabies virus-based vaccine YS-ON-001 for the treatment of hepatocellular carcinoma and pancreatic cancer [44]. Thus, TLR3 agonists may represent a suitable adjuvant for the design of DC vaccines against breast cancer.

Delivery of ICD inducers, such as SR-4835 (a CDK12/13-specific inhibitor), RIG-I agonists and chemotherapeutics, as well as photothermal agents, with synthetic nanoparticles, has been confirmed to have therapeutic potential in several preclinical models of breast cancer [45-47]. Although Texs play a major role in tumor immune evasion and growth, they can be manipulated and have tumor-homing properties, as well as low in vivo toxicity and immunogenicity [48]. Therefore, we loaded Hiltonol and ELANE into breast cancer cell-derived exosomes distinct from previous synthetic nanocarriers. Moreover, ELANE is a serine protease released by human neutrophils that selectively kills cancer cells and has no toxicity to normal cells [30]. Although much work is necessary for systematic assessment of potential short-term and long-term toxicity, our small-scale pilot toxicity study, together with the safety data from clinical trials of autologous/allogenic exosomes, can alleviate safety concerns about our HELA-Exos.

Although exosome-based therapeutics hold great potential for treating multiple human diseases, the application of exosomes in drug delivery has been limited because producing sufficient quantities for in vivo use is technically challenging [49]. Currently, electroporation is the most widely used technique for exosome loading with exogenous cargo [50]. However, this approach is poorly suited for loading macromolecular proteins and inserting large nucleic acids. In the future, newly constructed approaches that overcome these shortcomings, such as 
"exosomes for protein loading via optically reversible protein-protein interactions" (EXPLORs) [51] and cellular nanoporation [49], should be introduced for loading exogenous cargoes into exosomes.

Autologous DC vaccines are the most investigated approaches and have been extensively investigated in more than 200 completed clinical trials but have not yet provided robust clinical benefits in large patient populations $[10,52-54]$. The potential reason may be that this approach involves the ex vivo differentiation, antigen loading and maturation of autologous DCs in a controlled environment and reinfusion into patients. Thus, it is often difficult to standardize and scale up the precise conditions, such as the DC source (monocytes, CD $34^{+}$precursors or other subsets), the composition of the maturation cocktail, the nature of the tumor antigen (TAA or tissuespecific antigens), and the route and dose of DC vaccination [55]. Moreover, the cost is higher than that of other approaches due to the need for personalized cell therapy products. Compared to the preparation of autologous DC vaccines, it is conceivable that targeted delivery of ICD inducers and adjuvants into tumor cells to activate DCs in situ can be generalized to many other types of cancers.

In summary, we developed and optimized a formulation of tumor cell-specific exosomes (HELA-Exos) to specifically transfer the TLR3 agonist Hiltonol and the ICD inducer ELANE into tumor cells. This strategy enhances the immunogenicity of TNBC cells and indirectly activates tumor-infiltrating $\mathrm{CDC} 1 \mathrm{~s}$ in situ in both a mouse xenograft model and patient-derived tumor organoids. Beyond breast cancer, tumor-derived exosome-based delivery of ICD inducers and controllable adjuvants to tumor cells can be extended to various types of cancers.

\section{Methods}

\section{Culture of cell lines}

MDA-MB-231/luc cells (luciferase-expressing cells) were purchased from Guan\&Dao Biological Technology Ltd. MDA-MB 231, MDA-MB-231/luc, and MDA-MB-435S cells were grown in Leibovitz's L-15 (HyClone) supplemented with $10 \% \mathrm{FBS}$ at $37{ }^{\circ} \mathrm{C}$ in a suitable incubator without $\mathrm{CO}_{2}$. MCF10A cells were grown in MEGM ${ }^{\mathrm{TM}}$ BulletKit $^{\mathrm{TM}}$ (Lonza) supplemented with $100 \mathrm{ng} / \mathrm{mL}$ cholera toxin. BGC823 cells, T24 cells, A549 cells, MCF7 cells, and SKBR3 cells were grown in DMEM (HyClone), THP1 cells were grown in RPMI 1640 (HyClone), the media were supplemented with $10 \%$ FBS. The cells were grown at $37{ }^{\circ} \mathrm{C}$ in the presence of $5 \% \mathrm{CO}_{2}$. MDA-MB 231, MDA-MB-435S, MCF10A, A549, MCF7, and SKBR3 cells were kindly provided by Professor Shi Liu (Wuhan University). BGC823, T24, and THP1 cells were kindly provided by Professor Shaoping Liu (Zhongnan Hospital of Wuhan University).

\section{Reverse transcription qPCR analysis}

RT-qPCR was performed to detect the mRNA levels of $\alpha$-LA in cells. Total RNA was extracted from the cells using TRIzol (Invitrogen, Cat: 10,296,010) according to the manufacturer's instructions. The RNA concentration and purity were measured with a NanoDrop One trace spectrophotometer (Thermo Fisher Scientific). Reverse transcription of RNA was performed with ReverTra Ace qPCR RT Master Mix (TOYOBO, Cat: FSQ-201). qPCR was performed on a StepOnePlus Real-Time PCR System (Applied Biosystems) by using a standard SYBR green assay protocol. The specificity of the $\mathrm{qPCR}$ products and the data were analyzed using the sequence detection software supplied with StepOne Software (Applied Biosystems). The qPCR primers used in this experiment are given in Supplementary Table 2.

\section{Cell transfection}

The human $\alpha$-LA full-length coding sequence was cloned from total RNA isolated from MDA-MB-231 cells and cloned into the lentiviral expression vector $\mathrm{pCDH}-\mathrm{CMV}$ puro (System Biosciences, USA). Human 293 T cells were seeded in a $6 \mathrm{~cm}$ Petri dish for $24 \mathrm{~h}$, followed by cotransfection with pCDH-CMV-puro- $\alpha$-LA, pMD2.G and psPAX2 plasmids at a ratio of 2:1:1 with DNA transfection reagent (Neofect, USA, Cat: TF201201). Viruses were harvested and titred $48 \mathrm{~h}$ later and used for subsequent MDAMB-231 cell infection. MDA-MB-231 cells were seeded in 12-well plates and infected with $\alpha$-LA-expressing lentivirus. The infection was repeated 3 times every $18 \mathrm{~h}$, followed by puromycin selection $(2 \mu \mathrm{g} / \mathrm{mL})$. Exosomes were harvested from $\alpha$-LA/MDA-MB-231 cells.

\section{Western blotting}

Various amounts of protein were prepared from cell lysates or exosomes. The concentration of protein was detected with a BCA Protein Assay Kit (Beyotime Biotechnology, Cat: P0012S). Protein samples were separated using sodium dodecyl sulfate-polyacrylamide gel electrophoresis (SDS-PAGE) and transferred onto nitrocellulose membranes (EMD Millipore). The membranes were blocked with $5 \%$ nonfat milk at room temperature for $2 \mathrm{~h}$ and incubated overnight with the indicated primary antibody. Secondary antibody was added and incubated for $1 \mathrm{~h}$ at $37^{\circ} \mathrm{C}$. Finally, the blots were detected with an enhanced chemiluminescence kit (Meilunbio, Cat: MA0186) and analyzed with VisionWorksLS Software. The antibodies used for Western blotting are presented in Supplementary Table 3.

\section{Exosome preparation}

Cell culture medium was sequentially centrifuged at $300 \mathrm{~g}$ for $10 \mathrm{~min}, 2,000 \mathrm{~g}$ for $10 \mathrm{~min}$, and 10,000 $\mathrm{g}$ for 
$30 \mathrm{~min}$. The supernatant was collected and filtered with a $0.22 \mu \mathrm{m}$ filter, followed by ultracentrifugation at $100,000 \mathrm{~g}$ for $1 \mathrm{~h}$ to pellet exosomes. Exosome pellets were washed in a large volume of PBS and recovered by centrifugation at $100,000 \mathrm{~g}$ for $1 \mathrm{~h}$. All centrifugation steps were carried out at $4{ }^{\circ} \mathrm{C}$. The total protein concentration of exosomes was quantified with a BCA Protein Assay Kit (Beyotime Biotechnology, Cat: P0012S).

\section{Preparation of HELA-Exos}

ELANE and Hiltonol were loaded into exosomes to fabricate the vaccine by using an electroporation system (Bio-Rad Gene Pluser Xcell). ELANE was labeled with FITC with the EZLabel ${ }^{\mathrm{TM}}$ Protein FITC Labeling Kit (Biovision, Cat: K832-5*1EA), and Hiltonol was labeled with CX-Rhodamine with the Label $\mathrm{IT}^{\mathrm{TM}}$ Nucleic Acid Labeling Kit, CX-Rhodamine (Mirus Bio, Cat: MIR3100) according to the manufacturer's instructions. FITC-labeled ELANE or CX-rhodamine-labeled Hiltonol was dispersed in pure water at various concentrations, and the fluorescence intensity was measured at $519 \mathrm{~nm}$ or $597 \mathrm{~nm}$ to generate a fluorescence standard curve for the empirical optimization of the electroporation protocol. A series of electroporation parameters were set, including capacitance, voltage and buffer volume. Exosomes, ELANE, and Hiltonol were mixed in electroporation buffer and electroporated in a $4 \mathrm{~mm}$ cuvette. After electroporation, the exosomes were spun down at 100,000 g for $1 \mathrm{~h}$. Then, they were lysed in pure water, and fluorescence was assayed at $519 \mathrm{~nm}$ and $597 \mathrm{~nm}$ on a fluorescent plate reader for quantification of ELANE and Hiltonol based on the fluorescence standard curve, ultimately yielding the optimal electroporation parameters. The optimal loading efficiencies of ELANE and Hiltonol were $12.45 \%$ and $21.31 \%$, respectively.

Finally, vaccines (HLA-Exos, ELA-Exos, and HELAExos) were fabricated with electroporation parameters of $400 \mathrm{~V}$ and $150 \mu \mathrm{F}$ in $300 \mu \mathrm{L}$ of buffer. After electroporation, exosomes were washed two times in PBS with 100,000 g, centrifuged for $1 \mathrm{~h}$ and resuspended in medium for follow-up research. The loaded ELANE or Hiltonol was quantified according to the loading efficiency.

\section{Nanoscale flow cytometry of exosomes}

The exosomes loaded with FITC-labeled ELANE and CX-Rhodamine-labeled Hiltonol were analyzed using a A50-Micro Plus Nanoscale Flow Cytometer (Apogee FlowSystems Inc.) equipped with $70 \mathrm{~mW} 405 \mathrm{~nm}$ (violet), $53 \mathrm{~mW} 488 \mathrm{~nm}$ (blue) and $73 \mathrm{~mW} 639 \mathrm{~nm}$ (red) lasers. The parameters in the control panel were set to a sheath pressure of 150 mbar and a number of flush cycles of 3 . A sample flow rate of $1.5 \mu \mathrm{L} / \mathrm{min}$ was used for all measurements, and the time of acquisition was held constant for all samples at $60 \mathrm{~s}$ to yield enough events. An illumination wavelength of $405 \mathrm{~nm}(70 \mathrm{~mW})$ was used to detect scattered light. Before sample analysis, calibration of the flow cytometer was performed using silica nanoparticles (Apogee FlowSystems Inc.). Silica nanoparticles and fluorescent polystyrene nanoparticles were used to evaluate the sensitivity and resolution of flow cytometry scattered light and fluorescence. The data were analyzed using FlowJo Software.

\section{Characterization of the morphology and diameter of HELA-Exos}

The morphology of all the nanoparticles was characterized using transmission electron microscopy (HT7700, Hitachi). Negative staining TEM images were recorded after staining the samples with uranyl acetate (3\%). The diameters of all the nanoparticles were analyzed by dynamic light scattering (DLS) with a particle sizer (Zetasizer Nano ZS, Malvern). For the stability analysis, HELA-Exos were dispersed in PBS ( $\mathrm{pH} 7.4$ ) and 10\% FBS for one week, and the diameter was recorded every day.

\section{Targeting of HELA-Exos}

Exosomes were labeled with DiI (Beyotime Biotechnology, Cat: C1991S) according to the manufacturer's instructions. Briefly, purified exosomes from MDAMB-231 or $\alpha$-LA/MDA-MB-231 cells were incubated in the presence of $5 \mathrm{mM}$ DiI for $15 \mathrm{~min}$ at $37{ }^{\circ} \mathrm{C}$ and then ultracentrifuged at $100,000 \mathrm{~g}$ for $1 \mathrm{~h}$ to remove the unbound dye. After being washed twice in PBS with centrifugation at 100,000 $\mathrm{g}$ for $1 \mathrm{~h}$, Texs-DiI and LA-ExosDiI were collected, and LA-Exos-DiI was electroporated to prepare HELA-Exos-DiI.

MDA-MB-231 cells were collected and labeled with CFSE (Beyotime Biotechnology, Cat: C0051) according to the manufacturer's instructions. The PBMC fraction was isolated from peripheral blood by Ficoll-Paque density gradient separation and mixed with MDA-MB-231 cells at a ratio of 5:1 to establish a coculture model. The coculture model was incubated with Texs-DiI or HELA-ExosDiI for $2 \mathrm{~h}$ at $37^{\circ} \mathrm{C}$ and then stained with a Pacific Blue anti-human CD45 antibody (Biolegend, Cat: 304,022) for $30 \mathrm{~min}$ at $4{ }^{\circ} \mathrm{C}$ to mark PBMCs. The targeting capacity of HELA-Exos to MDA-MB-231 cells was detected by flow cytometry (BD FACSAria III). For the CLSM assay, MDA-MB-231 cells or A549 cells were seeded in 12-well plates for $24 \mathrm{~h}$ and then coincubated with TexsDiI or HELA-Exos-DiI for $2 \mathrm{~h}$ at $37^{\circ} \mathrm{C}$. After rinsing with PBS twice and staining with DAPI (Sigma-Aldrich, Cat: 
D9542) for $15 \mathrm{~min}$, the fluorescence emission of DAPI and DiI was measured to determine the uptake efficiency of MDA-MB-231 cells or A549 cells.

\section{Preparation of human PBMC-derived DCs}

To prepare human PBMC-derived DCs, human monocytes were enriched by plastic adherence of PBMCs in a $100 \mathrm{~mm}$ Petri dish at $37{ }^{\circ} \mathrm{C}$ and $5 \% \mathrm{CO}_{2}$. After $2 \mathrm{~h}$ of incubation, the nonadherent cells were reserved to sort $\mathrm{CD}^{+} \mathrm{T}$ cells with a Naive $\mathrm{CD}^{+} \mathrm{T}$ Cell Isolation Kit (Miltenyi Biotec, Cat: 130-093-244) for subsequent experiments. The remaining adherent cells were cultured in medium containing $100 \mathrm{ng} / \mathrm{mL}$ human GM-CSF (PeproTech, Cat: AF-300-03-50UG) and $20 \mathrm{ng} / \mathrm{mL}$ human IL-4 (InvivoGen, Cat: rcyec-hil4). After $48 \mathrm{~h}$, the medium was refreshed, and $10 \mathrm{ng} / \mathrm{mL}$ TNF- $\alpha$ (InvivoGen, Cat: rcyc-htnfa) was added. On day 3 , the human DC suspension was harvested.

\section{Viability and ICD evaluation of cells treated with HELA-Exos in vitro}

Cancer cells (MDA-MB-231, MCF7, MDA-MB-435S, SKBR3) or noncancer cells (PBMCs, PBMC-derived DCs, MCF10A) were plated in complete growth media. Cells were washed with serum-free DMEM and treated with various vaccines (Texs, LA-Exos, HLA-Exos, ELA-Exos, and HELA-Exos; each vaccine dose contained or did not contain $3 \mu \mathrm{g}$ ELANE or $20 \mu \mathrm{g}$ Hiltonol) for $48 \mathrm{~h}$, and cell viability was assessed using several methods:

(1) Calcein-AM assay

Forty-eight hours after treatment, cells were incubated with calcein-AM (Invitrogen, $4 \mathrm{ng} / \mathrm{mL}$, Cat: C1430) and washed with serum-free DMEM. For representative images, the fluorescence emission of calcein-AM was collected by CLSM. For the quantitative detection of cell viability, fluorescence was measured at $495 \mathrm{~nm} / 516 \mathrm{~nm}$ using a SpectraMax Microplate Reader (Molecular Devices).

(2) Cleaved-CASP3 and cleaved-PARP1 analyses

Forty-eight hours after treatment, the cells were washed with PBS and lysed, and the concentration of protein was detected with a BCA Protein Assay Kit (Beyotime Biotechnology, Cat: P0012S). The cleaved CASP3/CASP3 and cleaved PARP1/PARP1 levels in treated MDA-MB-231 cells were measured by Western blotting.

(3) ATP and HMGB1 detection

Forty-eight hours after treatment, the cell culture supernatant of MDA-MB-231 cells was collected and evaluated using an ATP Assay Kit (Sigma-
Aldrich, Cat: 213-579-1) and a Human HMGB1 ELISA Kit (Genie, Cat: HUFI00660) according to the manufacturer's instructions.

(4) Cell immunofluorescence staining to detect CRT exposure

Forty-eight hours after treatment, the cells were washed with PBS and fixed with 4\% paraformaldehyde for $15 \mathrm{~min}$ at room temperature. Next, the cells were permeabilized with $0.1 \%$ Triton X-100 PBS for $10 \mathrm{~min}$ and cultured with blocking buffer containing $5 \%$ bovine serum albumin (BSA) for 1 hour at room temperature. Then, the cell samples were incubated overnight with an anti-calreticulin antibody at $4{ }^{\circ} \mathrm{C}$, washed three times with PBS, and incubated in secondary antibody for 1 hour at room temperature. Finally, the cells were stained with DAPI, washed with PBS, and imaged using a confocal laser scanning microscope (Leica).

\section{Evaluation of the immune activation ability of HELA-Exos in vitro}

MDA-MB-231 cells were collected by trypsin digestion and coculture with PBMC-derived DCs that were harvested as described previously at a ratio of 1:1. The cells were washed with serum-free DMEM and pretreated with medium, Hiltonol $(20 \mu \mathrm{g})$, or HELA-Exos (containing $3 \mu \mathrm{g}$ ELANE and $20 \mu \mathrm{g}$ Hiltonol) for $24 \mathrm{~h}$. Naive CD8 ${ }^{+}$ $\mathrm{T}$ cells that were sorted as described above were cultured in medium containing $150 \mathrm{U} / \mathrm{ml}$ recombinant human IL-2. Twenty-four hours after pretreatment, naive $\mathrm{CD} 8^{+}$ $\mathrm{T}$ cells were added to the coculture system, and coculture was continued for $48 \mathrm{~h}$. Forty-eight hours later, the cells were collected for flow cytometry analysis. To detect the specific killing efficacy of $\mathrm{CD}^{+} \mathrm{T}$ cells, $\mathrm{CD} 8^{+} \mathrm{T}$ cells were harvested and incubated with target cells (MDAMB-231, MCF7, A549, and MCF10A cells) at a ratio of 10:1 in 96-microwell plates at $37{ }^{\circ} \mathrm{C}$ and $5 \% \mathrm{CO}_{2}$ for $72 \mathrm{~h}$ as the reactive cell group. Concurrently, the pure $\mathrm{CD}^{+}$ $\mathrm{T}$ cell group, pure target cell control group and pure culture solution blank control group were established. In the last $4 \mathrm{~h}, 10 \mu \mathrm{L}$ CCK8 solution (Beyotime Biotechnology, Cat: C0039) was added. The OD value indicating the absorbance of enzyme-labeled cells was detected with an instrument at $570 \mathrm{~nm}$. The following formula was used to calculate the killing rate: killing rate $=[1-(\mathrm{OD}$ value of reactive cell well-OD value of effector cell well)/(OD value of target cell well)] $\times 100 \%$.

\section{Flow cytometry}

Antibodies for flow cytometry analysis were purchased from BioLegend. For the flow cytometry analysis of surface markers, the cells were stained on ice with 
fluorescence-conjugated antibodies for $30 \mathrm{~min}$ according to the manufacturer's instructions. For the staining of intracellular markers, such as granzyme B, the cells were treated with brefeldin A (InvivoGen, Cat: inhbfa) at $37{ }^{\circ} \mathrm{C}$ for $6 \mathrm{~h}$. Cells were stained with antibodies against surface markers and then fixed and permeabilized using an intracellular staining kit (BD Biosciences, Cat: $559,302,559,311)$ at $4{ }^{\circ} \mathrm{C}$ for $30 \mathrm{~min}$ and then stained with target intracellular antibody at $4{ }^{\circ} \mathrm{C}$ for $30 \mathrm{~min}$. Stained cells were finally evaluated by flow cytometry and analyzed using FlowJo Software. The antibodies used for flow cytometry are presented in Supplementary Table 3.

\section{Tumor-bearing mice and treatment}

$\mathrm{BALB} / \mathrm{c}$ mice were raised in the animal facility of the Experimental Animal Center, Zhongnan Hospital of Wuhan University, under sterile conditions in air-filtered containers. The establishment of xenograft human tumor models in immunocompetent mice was conducted according to a previous report [56]. For depletion of CD11c ${ }^{+}$DCs, mice were intraperitoneally injected with $200 \mu \mathrm{g}$ purified anti-mouse CD11c mAb (Biolegend, Cat: 117,302) the day before the tumor challenge, and the injections were repeated every 2 to 3 days. Depletion efficiency was confirmed by immunofluorescence assays and flow cytometry.

A total of $5.0 \times 10^{6} \mathrm{MDA}-\mathrm{MB}-231 /$ luc cells in a volume of $50 \mu \mathrm{L}$ were implanted into the \#4 mammary fat pad on Day -21. Cells were maintained on wet ice during implantation. Prior to treatment initiation on day 0 , the mice were randomly allocated to groups, and treatment began at a mean tumor burden of $100 \mathrm{~mm}^{3}$ (range of group means, 96-104 $\mathrm{mm}^{3}$ ). Medium, Hiltonol (60 $\mu \mathrm{g} /$ dose), or HELA-Exos (containing $12 \mu \mathrm{g}$ ELANE and $60 \mu \mathrm{g}$ Hiltonol/dose) were administered intravenously twice per week for 4 weeks.

\section{In vivo pharmacokinetics and biodistribution}

A total of $5.0 \times 10^{6} \mathrm{MDA}-\mathrm{MB}-231 / \mathrm{luc}$ cells in a volume of $50 \mu \mathrm{L}$ were implanted into the mammary fat pad. On 15 th day after the tumor inoculation, the mice were intravenous injected with medium, CX-Rhodamine-labeled Hiltonol $(60 \mu \mathrm{g} / \mathrm{dose})$, or HELA-Exos-DiI (containing $12 \mu \mathrm{g}$ ELANE and $60 \mu \mathrm{g}$ Hiltonol/dose). At different time points, in vivo fluorescence imaging was performed using Bruker Xtreme BI system. At different time points, $20 \mu \mathrm{L}$ blood were harvested from the tail veins. The harvested samples were diluted with $30 \mu \mathrm{L}$ PBS and the concentration of Hiltonol or HELA-Exos were determined by measuring the Mean Fluorescence Intensity (MFI) of CXRhodamine or DiI on a SpectraMax Microplate Reader (Molecular Devices). The serum of untreated mice was served as background control for subtraction.

\section{Monitoring of tumor growth and collection of tumor tissues}

To measure tumor growth, mice were imaged using an IVIS Spectrum In Vivo Imaging System. Mice were injected with $150 \mathrm{mg} / \mathrm{kg}$ D-luciferin intraperitoneally. Mice were placed in an imaging chamber, and under $1-2 \%$ isoflurane gas anesthesia, they were imaged in the supine position. Bioluminescence was measured and superimposed on photographic images of the mice. Images were analyzed using Living Image 4.3.1. The breast signal was calculated using fixed-volume regions of interest to estimate the tumor burden, and total flux was calculated.

During the above antitumor experiment in vivo, tumor volumes were measured 2 to 3 times a week using a caliper and calculated using the equation $\mathrm{V}$ $\left(\mathrm{mm}^{3}\right)=1 / 2 \times \mathrm{L} \times \mathrm{W}^{2}$, where $\mathrm{L}$ (length) is the largest diameter and W (width) is the smallest diameter. We obtained tumor growth curves of individual mice in various treatment groups.

Tumor-bearing mice were sacrificed at day 30 (1 day after the last administration) after treatment. Serum samples were collected from mice before sacrifice, and the tumor tissues, draining lymph nodes, and major organs (heart, lung, liver, spleen, and kidney) were harvested for further research. The levels of serum IL-6 (Biovision, Cat: K4144-100), IL-12 (Genie, Cat: MOFI01240) and TNF- $\alpha$ were evaluated with ELISA kits (Genie, Cat: MOFI00104). Major organs were stained with HE. The levels of serum alanine transaminase (ALT), aspartate aminotransferase (AST), blood urea nitrogen (BUN), and creatinine (CREA) were evaluated with a Liver or Renal Function Activity Assay Kit (Servicebio, Cat: GM1102).

\section{Tumor immunohistochemistry and immunofluorescence}

Tumor-bearing mice were sacrificed at day 30 (1 day after the last administration) after treatment. Tumor tissues and draining lymph nodes were fixed in $4 \%$ paraformaldehyde in PBS for $24 \mathrm{~h}$, embedded in paraffin blocks, and sectioned $(5 \mu \mathrm{m})$. Slides were stained with anti-mouse primary antibodies. Signals were developed using the Vectastain Elite ABC Universal plus kit, peroxidase (Vector Laboratories, Cat: PK-8200) or fluorescently labeled secondary antibodies. Cell nuclei were labeled with hematoxylin or DAPI. An Olympus IX81 fluorescence microscope was used to photograph representative peroxidase staining samples or fluorescence samples. The antibodies used for immunohistochemistry and immunofluorescence are presented in Supplementary Table 3.

\section{Tumor immune cell analysis by flow cytometry}

Tumor-bearing mice were sacrificed at day 30 (1 day after the last administration) after treatment. The tumor tissue 
was cut into small pieces to generate a single-cell suspension with a Mouse Tumor Dissociation Kit (Miltenyi Biotec, Cat: 130-096-730) and gentleMACS ${ }^{\mathrm{TM}}$ Dissociators (Miltenyi Biotec), as instructed in the manufacturer's protocol. If a visible red pellet was present after the last centrifugation of the digestion process, erythrocytes were lysed in $2 \mathrm{~mL}$ Red Blood Cell Lysis Solution (Miltenyi Biotec, Cat: 130-094-183) for $5 \mathrm{~min}$ at room temperature and centrifuged at $400 \mathrm{~g}$. A Dead Cell Removal Kit (Miltenyi Biotec, Cat: 130-090-101) was used to eliminate dead cells from tissue preparations. Cells were labeled with various antibodies and analyzed by flow cytometry. The specific killing efficacy of $\mathrm{CD}^{+} \mathrm{T}$ cells was determined with a CCK-8 kit.

\section{Breast cancer organoid culture}

Breast cancer organoids were established essentially as described in Norman Sachs et al. [57]. Tumor tissue derived from surgical resection was cut into small pieces to generate a single-cell suspension with a Human Tumor Dissociation Kit (Miltenyi Biotec, Cat: 130-095929) and gentleMACS ${ }^{\mathrm{TM}}$ Dissociators (Miltenyi Biotec), as instructed in the manufacturer's protocol. If a visible red pellet was present after the last centrifugation of the digestion process, erythrocytes were lysed in $2 \mathrm{~mL}$ Red Blood Cell Lysis Solution (Miltenyi Biotec, Cat: 130-094183) for $5 \mathrm{~min}$ at room temperature and centrifuged at 400 g. A Dead Cell Removal Kit (Miltenyi Biotec, Cat: 130-090-101) was used to eliminate dead cells from tissue preparations. The cell pellet was resuspended in $10 \mathrm{mg} / \mathrm{mL}$ cold Cultrex Reduced Growth Factor Basement Membrane Matrix, Type 2 (BME 2) (Trevigen, Cat: 3533-001-02), and $40 \mu \mathrm{L}$ drops of BME-cell suspension were allowed to solidify on prewarmed 24-well suspension culture plates at $37{ }^{\circ} \mathrm{C}$ for $20 \mathrm{~min}$. Upon completion of gelation, $400 \mu \mathrm{L}$ of human breast cancer organoid medium was added to each well, and the plates were transferred to humidified $37{ }^{\circ} \mathrm{C}, 5 \% \mathrm{CO}_{2}$ incubators. The medium was changed every 4 days. In the first two weeks of organoid culture, primocin (InvivoGen, Cat: ant-pm-05) was added to prevent microbial contamination. The human breast cancer organoid medium recipe is provided in Supplementary Table 4.

\section{Organoid-PBMC coculture system}

The organoid-PBMC coculture system was established according to the protocol provided by Krijn K. Dijkstra et al. [58]. The $\mathrm{T}$ cell medium was composed of RPMI 1640 supplemented with $2 \mathrm{mM}$ Ultraglutamine I, 1\% penicillin/streptomycin and $10 \%$ male human $\mathrm{AB}$ serum (SeraCare, Cat: 1810-0001). Tumor organoids were dissociated into single cells with TrypLE Express and resuspended in $\mathrm{T}$ cell medium. PBMCs were seeded at a density of $10^{5}$ cells/well, stimulated with single celldissociated organoids at a 20:1 effector:target ratio in the presence of $150 \mathrm{U} / \mathrm{ml}$ recombinant human IL-2 (PeproTech, Cat: 200-02), and cocultured for 2 weeks in T cell medium. The coculture system was treated with medium, Hiltonol $(20 \mu \mathrm{g} / \mathrm{dose}$ ), or HELA-Exos (containing $3 \mu \mathrm{g}$ ELANE and $20 \mu \mathrm{g}$ Hiltonol/dose). Half of the medium, including IL-2, Hiltonol or HELA-Exos, was replaced 2 to 3 times per week. Every week, the PBMCs were restimulated with fresh tumor organoids.

\section{Evaluation of the immune activation and antitumor efficacy of HELA-Exos in a coculture system}

PBMCs were cocultured with autologous tumor organoids derived from breast cancer for 2 weeks and administered medium, Hiltonol or HELA-Exos. After two weeks of coculture, immune infiltration in the organoids was evaluated by flow cytometry and immunofluorescence, and the growth-inhibitory effects of HELA-Exos on organoids were evaluated via a cell viability assay and live/dead cell staining.

For flow cytometry, organoids were dissociated into single cells using TrypLE Express, and all the cells were collected for flow cytometry analysis. For immunofluorescence imaging, organoids were removed from the coculture system on day 14 and fixed with $4 \%$ paraformaldehyde for $30 \mathrm{~min}$. After fixation, the spheroids were transferred to tissue culture dishes and embedded in $4 \%$ agarose. Pre-embedded agarose spheroids were embedded in paraffin after isopropanol and acetone dehydration. Four-micrometer-thick sections were cut and adhered to poly-L-lysine-coated glass slides. The sections were incubated with the primary antibodies in a humidified chamber at $4{ }^{\circ} \mathrm{C}$ overnight. Then, the sections were incubated for $1 \mathrm{~h}$ with secondary antibodies. The cells were counterstained with DAPI for $5 \mathrm{~min}$. The sections were imaged to observe fluorescence at $488 \mathrm{~nm}, 594 \mathrm{~nm}$, and $380 \mathrm{~nm}$ with an Olympus IX81 microscope. The antibodies used for immunofluorescence are presented in Supplementary Table 3.

The cell viability of organoids was evaluated with an MTS assay as instructed in the manufacturer's protocol. The MTS Cell Proliferation Colorimetric Assay Kit (Biovision, Cat: K300) was used, with quantification at $490 \mathrm{~nm}$ on a plate reader. A live-dead assay for the organoids was performed by exposing treated organoids to the Live-Dead Cell Staining Kit (Biovision, Cat: K501) solution as instructed in the manufacturer's protocol. The organoids were incubated in live-dead solution for $20 \mathrm{~min}$ at $37{ }^{\circ} \mathrm{C}$ and then imaged immediately by CLSM. 


\section{Statistical analysis}

All data are reported as the mean values \pm SDs. Statistical analyses were carried out using GraphPad Prism software version 8.0. Comparisons between two groups were performed using an unpaired two-tailed Student's $t$ test. One-way ANOVA was used for comparisons of more than two groups. $P<0.05$ was considered to indicate statistical significance.

\begin{abstract}
Abbreviations
DCs: Dendritic cells; HELA-Exos: Hiltonol-ELANE-a-LA-engineered exosomes; ICD: Immunogenic cell death; ELANE: Human neutrophil elastase; TLR3: Toll-like receptor 3; TNBC: Triple-negative breast cancer; TME: Tumor microenvironment; DAMPs: Damage-associated molecular patterns; CDC1s: Type 1 conventional dendritic cells; CDC2s: Type 2 conventional dendritic cells; PBMCs: Peripheral blood mononuclear cells; APCs: Antigen-presenting cells; TAAs: Tumor-associated antigens; Texs: Tumor-derived exosomes; $\mathbf{a}$-LA: a-Lactalbumin; Exos: Exosomes; PBS: Phosphate-buffered saline; FBS: Fetal bovine serum; CRT: Calreticulin; PDOs: Patient-derived tumor organoids; ER: Estrogen receptor; PR: Progesterone receptor; HER2: Human epidermal growth factor receptor 2; DLS: Dynamic light scattering; CLSM: Confocal laser scanning microscopy; MFI: Mean fluorescence intensity; CASP3: Caspase 3.
\end{abstract}

\section{Supplementary Information}

The online version contains supplementary material available at https://doi. org/10.1186/s12943-022-01515-x.

Additional file 1: Figure S1. Electroporation protocol optimization and targeting of HELA-EXOs. (A and B) FITC-labeled ELANE or CX-rhodaminelabeled Hiltonol dispersed in pure water at various concentrations was applied for calibration. The fluorescence standard curve of ELANE or Hiltonol was generated by measuring the fluorescence intensity at $519 \mathrm{~nm}$ and $597 \mathrm{~nm}$. (C and D) FITC-labeled ELANE and CX-Rhodamine-labeled Hiltonol were electroporated with exosomes in $200 \mu \mathrm{l}$ of buffer at the settings shown on the $x$-axis, and ELANE and Hiltonol loading was determined based on the fluorescence standard curve. The data are presented as the mean $\pm S D ; n=3$. (E) The cellular uptake of MDA-MB-231 cells or A549 cells after incubation with Texs-Dil or HELA-Exos-Dil for $2 \mathrm{~h}$ was evaluated by CLSM. Blue: DAPI. Red: Dil. Scale bar, $25 \mu \mathrm{m}$. CLSM: confocal laser scanning microscopy. Figure S2. Apoptosis analysis and specific cytotoxic activity of CD8+ T cells. (A) Gating strategy for analysis of MDA-MB-231 cells; the percentages of PI-positive cells were measured. (B) Apoptosis analysis. Images of western blots for cleaved CASP3/CASP3 and cleaved PARP1/PARP1 in MDA-MB-231 cells. (C) CD8+ T cells were harvested from various treatment groups and incubated with target cells (MDA-MB-231, MCF7, A549, and MCF10A cells), and cytotoxicity was detected with a CCK-8 kit. The data are presented as the mean \pm SD; $n=6$. $t$ test and one-way ANOVA were performed for statistical analysis (****: $P<0.0001$; *: $P<0.05$; ns: $P>0.05$ ). Figure S3. Fluorescence imaging, pharmacokinetic curves, and safety of HELA-Exos in vivo. (A) In vivo fluorescence imaging in orthotopic MDA-MB-231 tumor-bearing mice at 2, 12, and $24 \mathrm{~h}$ following injection with CX-Rhodamine-labeled Hiltonol and HELA-Exos-Dil. (B) In vivo pharmacokinetic curves of Hiltonol and HELA-Exos. (C) Pathological examination of the major organs (the lung, heart, liver, spleen, and kidney). The tissue sections were stained with H\&E. Scale bar, $100 \mu \mathrm{m}$. (D) The levels of the serum biochemical parameters ALT, AST, BUN, and CREA. The data are presented as the mean $\pm S D ; n=6$. One-way ANOVA was performed for statistical analysis (ns: $P>0.05$ ). Figure S4. APCs were recruited to the tumor microenvironment after HELA-Exo treatment. Balb/c mice with orthotopic breast cancer were sacrificed at day 30 after treatment initiation. Tumor-infiltrating DCs (CD11c+) and macrophages
(F4/80+) were detected by IF. Blue: DAPI; Green: F4/80; Red: CD11c. Scale bar, $25 \mu \mathrm{m}$. IF: immunofluorescence. Figure S5. Specific cytotoxic activity of CD8+ T cells from the tumor microenvironment of Balb/c mice with orthotopic breast cancer. Balb/c mice with orthotopic breast cancer were sacrificed at day 30 after treatment initiation. The tumor tissue was cut into small pieces to generate a single-cell suspension. CD8+ T cells were sorted and incubated with target cells (MDA-MB-231, MCF7, A549, and MCF10A cells), and cytotoxicity was detected with a CCK-8 kit. The data are presented as the mean $\pm \mathrm{SD} ; \mathrm{n}=6$. A t test and one-way ANOVA were performed for statistical analysis (****: $P<0.0001$; ${ }^{* *}: \mathrm{P}<0.01$; ns: $P>0.05$ ). Figure S6. Reduced systemic inflammatory toxicity of HELAExos. Balb/c mice with orthotopic breast cancer were sacrificed at day 30 after treatment initiation. The serum levels of the cytokines $I L-6, I L-12$, and TNF-a. The data are presented as the mean $\pm \mathrm{SD} ; \mathrm{n}=6$. A t test was performed for statistical analysis (****: $P<0.0001 ; n s: P>0.05)$. Figure $\mathbf{S 7}$. DC depletion efficiency in Balb/c mice and specific cytotoxic activity of CD8+T cells. (A) Gating strategy for the analysis of DCs; the percentages of CD11c+ DCs in the lymph nodes and spleen were measured. (B) DCdepleted or nondepleted Balb/c mice with orthotopic breast cancer were sacrificed at Day 30 after treatment initiation. The tumor tissue was cut into small pieces to generate a single-cell suspension. CD8+ T cells were sorted and incubated with target cells (MDA-MB-231, MCF7, A549, and MCF10A cells), and cytotoxicity was detected with a CCK-8 kit. The data are presented as the mean $\pm S D ; n=6$. A t test and one-way ANOVA were performed for statistical analysis ( ${ }^{* * *}: P<0.0001$; ${ }^{*}: P<0.05 ; n s: P>0.05$ ). Figure S8. Tumor organoids were established from tumor tissue, and PBMCs were isolated from peripheral blood before the start of coculture. On the day of coculture, organoids were isolated from Geltrex, dissociated into single cells and plated together with PBMCs in the presence of vaccines. After 1 week of coculture, PBMCs were re-stimulated with tumor cells from organoids. After two weeks of coculture, immune infiltration in organoids was evaluated by flow cytometry and IF, and the growthinhibitory effects in organoids of HELA-Exos were evaluated by a viability assay and live/dead cell staining.

Additional file 2: Table 1. Patient information for had established tumor organoids.

Additional file 3: Table 2. List of primers used.

Additional file 4: Table 3. List of antibodies for this study.

Additional file 5: Table 4. Human breast cancer organoids medium recipe.

\section{Acknowledgements}

Not applicable

\section{Authors' contributions}

Study conception and design: $L X H, Y R, C H Y$ and FBW. Data acquisition: $L X H$, $Y R$, XT, KZY, PQ, and JXH. Analysis and data interpretation: $L X H, W H L, Y H$ and $X G$. Drafting of the manuscript: $L X H$ and $C H Y$. Contributed to the article and approved the submitted version: All authors.

\section{Funding}

This work was supported by the Improvement Project for Theranostic ability on Difficulty Miscellaneous disease (Tumor) and the research fund from medical Sci-Tech innovation platform of Zhongnan Hospital (No. ZLYNXM202008 and PTXM2021001), the Medical Top-talented youth development project of Hubei Province and the Fundamental Research Funds for the Central Universities (No. 2042021 kf0227), and the Sixth Training Program Foundation for Wuhan Medical Talents by the Health Commission of Wuhan and the Natural Science Foundation of Wuhan Municipal Health Commission (No. WX18Q03).

\section{Availability of data and materials}

The original contributions presented in the study are included in the article/ Supplementary Material, further inquiries can be directed to the corresponding authors. 


\section{Declarations}

\section{Ethics approval and consent to participate}

This research was conducted in accordance with international guidelines and the ethical standards outlined in the Declaration of Helsinki. This study was approved by the Medical Ethics Committee of Zhongnan Hospital of Wuhan University (No. 2021052) and written informed consent was obtained from each patient for the use of their tissue/PBMCs samples. All animal experiments were conducted in compliance with the institutional guidelines of the Animal Experiment Center of University/Animal Biosafety Level-III laboratory for the Use of Animals. All animal procedures were approved by the Experimental Animal Welfare Ethics Committee of Zhongnan Hospital of Wuhan University.

\section{Consent for publication}

All authors give consent for the publication of the manuscript in Molecular Cancer.

\section{Competing interests}

The authors declare that the research was conducted in the absence of any commercial or financial relationships that could be construed as a potential conflict of interest.

\section{Author details}

'Department of Laboratory Medicine, Zhongnan Hospital of Wuhan University, No.169 Donghu Road, Wuhan, Wuchang District, China. ${ }^{2}$ Center for Single-Cell Omics and Tumor Liquid Biopsy, Zhongnan Hospital of Wuhan University, Wuhan, China. ${ }^{3}$ Department of Thyroid and Breast Surgery, Hubei No. 3 People's Hospital of Jianghan University, Wuhan, China. ${ }^{4}$ Department of Thyroid and Breast Surgery, Zhongnan Hospital of Wuhan University, Wuhan, China. ${ }^{5}$ Medical Research Center for Structural Biology, School of Basic Medical Sciences, Wuhan University, Wuhan, China. ${ }^{6}$ Animal Experiment Center, Zhongnan Hospital of Wuhan University, Wuhan, China. ${ }^{7}$ Department of Laboratory Medicine, Wuhan Children's Hospital, Tongji Medical College, Huazhong University of Science and Technology, Wuhan, China. ${ }^{8}$ Wuhan Research Center for Infectious Diseases and Cancer, Chinese Academy of Medical Sciences, Wuhan, China.

Received: 22 November 2021 Accepted: 19 January 2022

Published online: 11 February 2022

\section{References}

1. Wculek SK, Cueto FJ, Mujal AM, Melero I, Krummel MF, Sancho D. Dendritic cells in cancer immunology and immunotherapy. Nat Rev Immunol. 2020;20:7-24.

2. Andtbacka RHI, Kaufman HL, Collichio F, Amatruda T, Senzer N, Chesney J, et al. Talimogene Laherparepvec Improves Durable Response Rate in Patients With Advanced Melanoma. J Clin Oncol. 2015;33:2780-8.

3. Kantoff PW, Higano CS, Shore ND, Berger ER, Small EJ, Penson DF, et al. Sipuleucel-T immunotherapy for castration-resistant prostate cancer. N Engl J Med. 2010;363:411-22.

4. Kreutz M, Tacken PJ, Figdor CG. Targeting dendritic cells-why bother? Blood. 2013;121:2836-44.

5. Wang H, Sobral MC, Zhang DKY, Cartwright AN, Li AW, Dellacherie MO, et al. Metabolic labeling and targeted modulation of dendritic cells. Nat Mater. 2020:19:1244-52

6. Dhodapkar MV, Sznol M, Zhao B, Wang D, Carvajal RD, Keohan ML, et al. Induction of antigen-specific immunity with a vaccine targeting NY-ESO-1 to the dendritic cell receptor DEC-205. Science translational medicine. 2014:6:232ra51.

7. Tacken PJ, de Vries IJ, Gijzen K, Joosten B, Wu D, Rother RP, et al. Effective induction of naive and recall $\mathrm{T}$-cell responses by targeting antigen to human dendritic cells via a humanized anti-DC-SIGN antibody. Blood. 2005;106:1278-85.

8. Kyi C, Roudko V, Sabado R, Saenger Y, Loging W, Mandeli J, et al. Therapeutic Immune Modulation against Solid Cancers with Intratumoral Poly-ICLC: A Pilot Trial. Clinical cancer research : an official journal of the American Association for Cancer Research. 2018;24:4937-48.
9. Melssen MM, Petroni GR, Chianese-Bullock KA, Wages NA, Grosh WW, Varhegyi $\mathrm{N}$, et al. A multipeptide vaccine plus toll-like receptor agonists LPS or polyICLC in combination with incomplete Freund's adjuvant in melanoma patients. J Immunother Cancer. 2019;7:163.

10. Harari A, Graciotti M, Bassani-Sternberg M, Kandalaft LE. Antitumour dendritic cell vaccination in a priming and boosting approach. Nat Rev Drug Discovery. 2020;19:635-52.

11. Galluzzi L, Buqué A, Kepp O, Zitvogel L, Kroemer G. Immunogenic cell death in cancer and infectious disease. Nat Rev Immunol. 2017;17:97-111.

12. Wang Z, Chen J, Hu J, Zhang H, Xu F, He W, et al. cGAS/STING axis mediates a topoisomerase II inhibitor-induced tumor immunogenicity. J Clin Investig. 2019;129:4850-62

13. Obeid M, Tesniere A, Ghiringhelli F, Fimia GM, Apetoh $L$, Perfettini J L, et al. Calreticulin exposure dictates the immunogenicity of cancer cell death. Nat Med. 2007;13:54-61.

14. Garg AD, Vandenberk L, Koks C, Verschuere T, Boon L, Van Gool SW, et al. Dendritic cell vaccines based on immunogenic cell death elicit danger signals and T cell-driven rejection of high-grade glioma. Science translational medicine. 2016;8:328ra27.

15. Chiang CL, Coukos G, Kandalaft LE. Whole Tumor Antigen Vaccines: Where Are We? Vaccines. 2015:3:344-72

16. Lin JH, Huffman AP, Wattenberg MM, Walter DM, Carpenter EL, Feldser DM, et al. Type 1 conventional dendritic cells are systemically dysregulated early in pancreatic carcinogenesis. J Exp Med. 2020;217:e20190673.

17. Salmon H, Idoyaga J, Rahman A, Leboeuf M, Remark R, Jordan S, et al. Expansion and Activation of CD103(+) Dendritic Cell Progenitors at the Tumor Site Enhances Tumor Responses to Therapeutic PD-L1 and BRAF Inhibition. Immunity. 2016;44:924-38.

18. Rodríguez-Ruiz ME, Perez-Gracia JL, Rodríguez I, Alfaro C, Oñate C, Pérez $G$, et al. Combined immunotherapy encompassing intratumoral polyICLC, dendritic-cell vaccination and radiotherapy in advanced cancer patients. Annals of oncology : official journal of the European Society for Medical Oncology. 2018;29:1312-9.

19. Enamorado M, Iborra S, Priego E, Cueto FJ, Quintana JA, MartínezCano S, et al. Enhanced anti-tumour immunity requires the interplay between resident and circulating memory CD8(+) T cells. Nat Commun. 2017:8:16073

20. Segura E, Durand M, Amigorena S. Similar antigen cross-presentation capacity and phagocytic functions in all freshly isolated human lymphoid organ-resident dendritic cells. J Exp Med. 2013;210:1035-47.

21. Böttcher JP, Bonavita E, Chakravarty P, Blees H, Cabeza-Cabrerizo M, Sammicheli S, et al. NK Cells Stimulate Recruitment of CDC1 into the Tumor Microenvironment Promoting Cancer Immune Control. Cell. 2018;172:1022-37.e14.

22. Ferris ST, Durai $V$, Wu R, Theisen DJ, Ward JP, Bern MD, et al. CDC1 prime and are licensed by CD4(+)T cells to induce anti-tumour immunity. Nature. 2020;584:624-9.

23. Mathieu M, Martin-Jaular L, Lavieu G, Théry C. Specificities of secretion and uptake of exosomes and other extracellular vesicles for cell-to-cell communication. Nat Cell Biol. 2019;21:9-17.

24. Choi JS, Cho WL, Choi YJ, Kim JD, Park HA, Kim SY, et al. Functional recovery in photo-damaged human dermal fibroblasts by human adiposederived stem cell extracellular vesicles. Journal of extracellular vesicles. 2019;8:1565885.

25. Nassar W, El-Ansary M, Sabry D, Mostafa MA, Fayad T, Kotb E, et al. Umbilical cord mesenchymal stem cells derived extracellular vesicles can safely ameliorate the progression of chronic kidney diseases. Biomaterials research. 2016;20:21.

26. Besse B, Charrier M, Lapierre V, Dansin E, Lantz O, Planchard D, et al. Dendritic cell-derived exosomes as maintenance immunotherapy after first line chemotherapy in NSCLC. Oncoimmunology. 2016;5:e1071008.

27. Dai S, Wei D, Wu Z, Zhou X, Wei X, Huang H, et al. Phase I clinical trial of autologous ascites-derived exosomes combined with GM-CSF for colorectal cancer. Molecular therapy : the journal of the American Society of Gene Therapy. 2008;16:782-90.

28. Sengupta V, Sengupta S, Lazo A, Woods P, Nolan A, Bremer N. Exosomes Derived from Bone Marrow Mesenchymal Stem Cells as Treatment for Severe COVID-19. Stem cells and development. 2020;29:747-54. 
29. Hadla M, Palazzolo S, Corona G, Caligiuri I, Canzonieri V, Toffoli G, et al. Exosomes increase the therapeutic index of doxorubicin in breast and ovarian cancer mouse models. Nanomedicine (Lond). 2016;11:2431-41.

30. Cui C, Chakraborty K, Tang XA, Zhou G, Schoenfelt KQ, Becker KM, et al. Neutrophil elastase selectively kills cancer cells and attenuates tumorigenesis. Cell. 2021;184:3163-77.e21.

31. Jaini R, Kesaraju P, Johnson JM, Altuntas CZ, Jane-Wit D, Tuohy VK. An autoimmune-mediated strategy for prophylactic breast cancer vaccination. Nat Med. 2010;16:799-803.

32. Yang J, Wang T, Zhao L, Rajasekhar VK, Joshi S, Andreou C, et al. Gold/ alpha-lactalbumin nanoprobes for the imaging and treatment of breast cancer. Nature biomedical engineering. 2020;4:686-703.

33. Couch FJ, Hart SN, Sharma P, Toland AE, Wang X, Miron P, et al. Inherited Mutations in 17 Breast Cancer Susceptibility Genes Among a Large TripleNegative Breast Cancer Cohort Unselected for Family History of Breast Cancer. J Clin Oncol. 2014;33:304-11.

34. Dent R, Trudeau M, Pritchard Kl, Hanna WM, Kahn HK, Sawka CA, et al. Triple-Negative Breast Cancer: Clinical Features and Patterns of Recurrence. Clin Cancer Res. 2007;13:4429.

35. Mattiuz R, Brousse C, Ambrosini M, Cancel JC, Bessou G, Mussard J, et al. Type 1 conventional dendritic cells and interferons are required for spontaneous CD4(+) and CD8(+) T-cell protective responses to breast cancer. Clinical \& translational immunology. 2021;10:e1305.

36. Cattaneo CM, Dijkstra KK, Fanchi LF, Kelderman S, Kaing S, van Rooij N, et al. Tumor organoid-T-cell coculture systems. Nat Protoc. 2020;15:15-39.

37. Denkert C, Liedtke C, Tutt A, von Minckwitz G. Molecular alterations in triple-negative breast cancer-the road to new treatment strategies. Lancet (London, England). 2017;389:2430-42.

38. Yin L, Duan JJ, Bian XW, Yu SC. Triple-negative breast cancer molecular subtyping and treatment progress. Breast cancer research : BCR. 2020;22:61.

39. Vassilaros S, Tsibanis A, Tsikkinis A, Pietersz GA, McKenzie IF, Apostolopoulos V. Up to 15-year clinical follow-up of a pilot Phase III immunotherapy study in stage II breast cancer patients using oxidized mannan-MUC1. Immunotherapy. 2013;5:1177-82.

40. Burke EE, Kodumudi K, Ramamoorthi G, Czerniecki BJ. Vaccine Therapies for Breast Cancer. Surg Oncol Clin N Am. 2019;28:353-67.

41. McDonald KA, Kawaguchi T, Qi Q, Peng X, Asaoka M, Young J, et al. Tumor Heterogeneity Correlates with Less Immune Response and Worse Survival in Breast Cancer Patients. Ann Surg Oncol. 2019;26:2191-9.

42. Hubert M, Gobbini E, Couillault C, Manh TV, Doffin AC, Berthet J, et al. IFNIII is selectively produced by CDC1 and predicts good clinical outcome in breast cancer. Science immunology. 2020;5:eaav3942.

43. Roselli E, Araya P, Núñez NG, Gatti G, Graziano F, Sedlik C, et al. TLR3 Activation of Intratumoral CD103(+) Dendritic Cells Modifies the Tumor Infiltrate Conferring Anti-tumor Immunity. Front Immunol. 2019;10:503.

44. Goyvaerts C, Breckpot K. The Journey of in vivo Virus Engineered Dendritic Cells From Bench to Bedside: A Bumpy Road. Front Immunol. 2018;9:2052.

45. Li Y, Zhang H, Li Q, Zou P, Huang X, Wu C, et al. CDK12/13 inhibition induces immunogenic cell death and enhances anti-PD-1 anticancer activity in breast cancer. Cancer Lett. 2020;495:12-21.

46. Elion DL, Jacobson ME, Hicks DJ, Rahman B, Sanchez V, Gonzales-Ericsson PI, et al. Therapeutically Active RIG-I Agonist Induces Immunogenic Tumor Cell Killing in Breast Cancers. Can Res. 2018;78:6183-95.

47. Li Y, Liu X, Pan W, Li N, Tang B. Photothermal therapy-induced immunogenic cell death based on natural melanin nanoparticles against breast cancer. Chem Commun (Camb). 2020;56:1389-92.

48. Taghikhani A, Farzaneh F, Sharifzad F, Mardpour S, Ebrahimi M, Hassan ZM. Engineered Tumor-Derived Extracellular Vesicles: Potentials in Cancer Immunotherapy. Front Immunol. 2020;11:221.

49. Yang Z, Shi J, Xie J, Wang Y, Sun J, Liu T, et al. Large-scale generation of functional mRNA-encapsulating exosomes via cellular nanoporation. Nature biomedical engineering. 2020;4:69-83.

50. Familtseva A, Jeremic N, Tyagi SC. Exosomes: cell-created drug delivery systems. Mol Cell Biochem. 2019;459:1-6.

51. Yim N, Ryu SW, Choi K, Lee KR, Lee S, Choi H, et al. Exosome engineering for efficient intracellular delivery of soluble proteins using optically reversible protein-protein interaction module. Nat Commun. 2016;7:12277.
52. Garg AD, Coulie PG, Van den Eynde BJ, Agostinis P. Integrating Next-Generation Dendritic Cell Vaccines into the Current Cancer Immunotherapy Landscape. Trends Immunol. 2017:38:577-93.

53. Bol KF, Schreibelt G, Gerritsen WR, de Vries IJ, Figdor CG. Dendritic CellBased Immunotherapy: State of the Art and Beyond. Clinical cancer research : an official journal of the American Association for Cancer Research. 2016:22:1897-906.

54. Melero I, Gaudernack G, Gerritsen W, Huber C, Parmiani G, Scholl S, et al. Therapeutic vaccines for cancer: an overview of clinical trials. Nat Rev Clin Oncol. 2014;11:509-24.

55. Mastelic-Gavillet B, Balint K, Boudousquie C, Gannon PO, Kandalaft LE. Personalized Dendritic Cell Vaccines-Recent Breakthroughs and Encouraging Clinical Results. Front Immunol. 2019;10:766

56. Basel MT, Narayanan S, Ganta C, Shreshta TB, Marquez A, Pyle M, et al. Developing a xenograft human tumor model in immunocompetent mice. Cancer Lett. 2018:412:256-63.

57. Sachs N, de Ligt J, Kopper O, Gogola E, Bounova G, Weeber F, et al. A Living Biobank of Breast Cancer Organoids Captures Disease Heterogeneity. Cell. 2018;172:373-86.e10.

58. Dijkstra KK, Cattaneo CM, Weeber F, Chalabi M, van de Haar J, Fanchi LF, et al. Generation of Tumor-Reactive T Cells by Co-culture of Peripheral Blood Lymphocytes and Tumor Organoids. Cell. 2018;174:1586-98.e12.

\section{Publisher's Note}

Springer Nature remains neutral with regard to jurisdictional claims in published maps and institutional affiliations.

Ready to submit your research? Choose BMC and benefit from

- fast, convenient online submission

- thorough peer review by experienced researchers in your field

- rapid publication on acceptance

- support for research data, including large and complex data types

- gold Open Access which fosters wider collaboration and increased citations

- maximum visibility for your research: over 100M website views per year

At BMC, research is always in progress.

Learn more biomedcentral.com/submissions 